\title{
高度正则图的全着色
}

\section{谢德政 ${ }^{*}$, 杨万年}

重庆大学数理学院, 重庆 400044

*E-mail: xdzwp568@ctbu.edu.cn

收稿日期: 2007-10-16; 接受日期: 2008-07-15

重庆市科委自然科学基金计划 (批准号: CSTC, 2007BB2123) 资助项目

摘要 一个图 $G$ 的全色数 $\chi_{T}(G)$ 是对 $G$ 的边和顶点着色的最小数, 使得相关联或相邻 元素着不同色. 证明了如果 $G$ 是正则图并且

$$
d(G) \geqslant \frac{2}{3}|V(G)|+\frac{23}{6},
$$

这里 $d(G)$ 表示在 $G$ 中顶点的度, 则 $\chi_{T}(G) \leqslant d(G)+2$.

关键词全色数 全着色 全着色猜想

$\operatorname{MSC}(2000)$ 主题分类 $05 \mathrm{C} 15$

\section{1 引言}

本文中考虑的均为简单有限图. 设 $G$ 是一个图, 用 $V(G), E(G), \bar{G}, \delta(G), \Delta(G)$ 和 $\omega(G)$ 分别表示图 $G$ 的顶点集、边集、补图、最小度、最大度和分支数. 如果 $F \subset E(G)$, 则 $G-F$ 表示由图 $G$ 删除边集 $F$ 而得到的图. 如果 $E^{\prime} \subset E(\bar{G})$, 则 $G+E^{\prime}$ 是由图 $G$ 添加 $E^{\prime}$ 而生成 的图. 如果 $S \subset V(G)$ 则 $G[S]$ 表示由 $S$ 导出的 $G$ 的子图; 导出子图 $G[V(G) \backslash S]$ 记为 $G-S$. 如果 $A, B \subset V(G)$ 并且 $A \cap B=\emptyset$, 则 $G[A, B]$ 表示由 $A$ 和 $B$ 导出的 $G$ 的偶子图. $e_{G}(A, B)$ 表示图 $G[A, B]$ 中的边数. 如果 $G$ 是正则图, 则 $d(G)$ 表示在 $G$ 中顶点的度.

对于图 $G$, 函数 $\pi: V(G) \cup E(G) \rightarrow N$ 称为一个 (正常的) 全着色, 如果相邻或相关联的 两个元素着 $N$ 中的不相同的颜色. 图 $G$ 的全色数表示为 $\chi_{T}(G)$, 是指对于 $G$ 的所有的全着色 $\pi: V(G) \cup E(G) \rightarrow\{1, \ldots, k\}$ 中最小的正整数 $k$. 由全着色的定义, 显然 $\chi_{T}(G) \geqslant \Delta(G)+1$.

Behzad $^{[1]}$ 和 Vizing ${ }^{[2]}$ 各自独立的提出了以下猜想:

全着色猜想 (TCC) 对于任意的图 $G, \chi_{T}(G) \leqslant \Delta(G)+2$.

对于完全图和完全二部图, TCC 已被证明 ${ }^{[3,4]}$. Kostochka ${ }^{[5,6]}$ 和 Vijayaditya ${ }^{[7]}$ 证明了 $\Delta(G) \leqslant 5$ 时, TCC 成立. Borodin ${ }^{[8]}$ 证明了对于平面图 $G$ 并且 $\Delta(G) \geqslant 9$ 时, TCC 成 立. Yap ${ }^{[9]}$ 和 Andersen ${ }^{[10]}$ 证明了对于平面图 $G$ 并且 $\Delta(G)=8$ 时, TCC 成立. Sanders 和 Zhao ${ }^{[11]}$ 证明了平面图 $G$ 并且 $\Delta(G)=7$ 时, TCC 成立. 因此对于 $\Delta(G) \neq 6$ 的平面图, TCC 成立. 
对于高度图, Hilton 和 Hind ${ }^{[12]}$ 证明了对于图 $G$ 满足 $\Delta(G) \geqslant \frac{3}{4}|V(G)|$ 时, TCC 成立; Yap 等 [13] 证明了对于图 $G$ 满足 $\Delta(G) \geqslant|V(G)|-5$ 时, TCC 成立; Xie 和 $\mathrm{He}^{[14]}$ 证明了对 于偶数阶的图 $G$ 满足 $d(G) \geqslant \frac{2}{3}|V(G)|+\frac{23}{6}$ 时, TCC 成立. 关于全着色的其他的相关结果请 参见文献 [15-17]. 在本文中, 我们证明了如果 $G$ 是正则图并且满足 $d(G) \geqslant \frac{2}{3}|V(G)|+\frac{23}{6}$ 时, TCC 成立.

\section{2 预备结果}

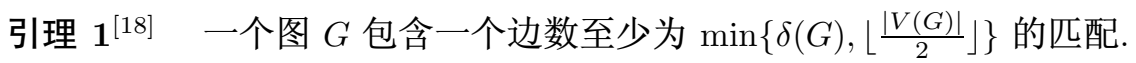

引理 2 设 $G$ 是一个 $n$ 阶的正则图并且

$$
\frac{2 n}{3}+\frac{7}{2} \leqslant d(G) \leqslant \frac{5 n}{6}-3,
$$

设 $n-3 \geqslant d(G)$ 并且 $k=n-d(G)-2$, 则存在 $V(G)$ 的一个子集 $\left\{x_{1}, y_{1}, z_{1}, \ldots, x_{k}, y_{k}, z_{k}\right\}$, 使得对于 $1 \leqslant i \leqslant k, x_{i} y_{i} \notin E(G)$, 并且 $y_{i} z_{i} \in E(G)$.

证明 由引理的假设, 得

$$
k=n-d(G)-2 \leqslant n-\left(\frac{2 n}{3}+\frac{7}{2}\right)-2=\frac{n}{3}-\frac{11}{2},
$$

则 $k<\delta(\bar{G})=n-d(G)-1 \leqslant \frac{n}{3}-\frac{9}{2}$. 因此, 由引理 1 , 存在 $V(G)$ 的一个子集 $\left\{x_{1}, y_{1}, \ldots, x_{k}, y_{k}\right\}$, 使得对于 $1 \leqslant i \leqslant k, x_{i} y_{i} \notin E(G)$. 设 $V_{1}=\left\{x_{1}, y_{1}, \ldots, x_{k}, y_{k}\right\}$. 由引理假设, 得

$$
\left|V_{1}\right|=2 k=2(n-d(G)-2) \geqslant 2\left(n-\left(\frac{5 n}{6}-3\right)-2\right)=\frac{n}{3}+2,
$$

因此, $\left|V(G) \backslash V_{1}\right| \leqslant n-\left(\frac{n}{3}+2\right)=\frac{2 n}{3}-2<d(G)$, 所以, $V(G) \backslash V_{1}$ 包含至少 1 个顶点, 记为 $z_{1}$, 使得 $z_{1}$ 与 $V_{1}$ 的至少 1 个顶点相邻. 假设 $u \in\left\{x_{i}, y_{i}\right\} \subset V_{1}$, 使得 $u z_{1} \in E(G)$. 如果 $u=x_{i}$, 分别用 $x_{i}$ 和 $y_{i}$ 替换 $y_{1}$ 和 $x_{1}$; 如果 $u=y_{i}$, 我们分别用 $x_{i}$ 和 $y_{i}$ 替换 $x_{1}$ 和 $y_{1}$, 则 $i=1$ 时 结论成立. 假设对于 $1 \leqslant i-1 \leqslant k-1$ 时结论成立. 由 (1) 式,

$$
\left|V(G) \backslash V_{1}\right|=n-2 k \geqslant n-2\left(\frac{n}{3}-\frac{11}{2}\right)=\frac{n}{3}+11>k .
$$

设 $V(G) \backslash V_{1}=V_{2}=\left\{z_{1}, \ldots, z_{i-1}, \ldots, z_{k}, \ldots, z_{l}\right\}$, 则 $l>k$. 设 $V_{3}=\left\{x_{i}, y_{i}, \ldots, x_{k}, y_{k}\right\}$ 且 $V_{4}=\left\{z_{i}, \ldots, z_{k}, \ldots, z_{l}\right\}$.

情形 1 若存在 $V_{4}$ 的一个顶点 $u$, 使得 $u$ 与 $V_{3}$ 的至少 1 个顶点相邻.

不失一般性, 假设 $u=z_{i}$ 且 $x_{j} z_{i} \in E(G)$ 或者 $y_{j} z_{i} \in E(G)$, 这里 $i \leqslant j \leqslant k$. 如果 $x_{j} z_{i} \in E(G)$, 则用 $y_{j}$ 和 $x_{j}$ 分别代替 $x_{i}$ 和 $y_{i}$. 如果, $y_{j} z_{i} \in E(G)$, 则用 $x_{j}$ 和 $y_{j}$ 分别代替 $x_{i}$ 和 $y_{i}$.

情形 2 若 $V_{4}$ 的顶点都不与 $V_{3}$ 的顶点相邻, 但 $V_{4}$ 包含至少两个顶点 $v$ 和 $w$, 使得 $v w \in E(G)$.

不失一般性, 假设 $v=z_{i}, w=z_{i+1}$, 则用 $z_{i+1}$ 替代 $y_{i}$.

情形 3 若 $V_{4}$ 的顶点都不与 $V_{3}$ 的顶点相邻, 且 $V_{4}$ 中任何两个顶点都不相邻.

设 $V_{5}=V(G) \backslash\left(V_{3} \cup V_{4}\right)$, 则 $V_{5}=\left\{x_{1}, y_{1}, z_{1}, \ldots, x_{i-1}, y_{i-1}, z_{i-1}\right\}$. 在此情形下, 因为 $d(G) \geqslant \frac{2 n}{3}+\frac{7}{2}$, 所以, $V_{5}$ 至少包含 1 个子集 $\left\{x_{j}, y_{j}, z_{j}\right\}$, 使得两个顶点 $z_{i}$ 和 $z_{i+1}$ 与 $\left\{x_{j}, y_{j}, z_{j}\right\}$ 中的至少两个顶点都相邻, 这里 $1 \leqslant j \leqslant i-1$, 否则, $d_{G}\left(z_{i}\right)+d_{G}\left(z_{i+1}\right) \leqslant 4(i-1) \leqslant 4 k$, 因 
为 $G$ 是一个正则图, 由 (1) 式, 有 $d_{G}\left(z_{i}\right) \leqslant \frac{2 n}{3}-11$, 这与引理的假设矛盾. 不失一般性, 假设 $x_{j} z_{i} \in E(G)$ 和 $y_{j} z_{i+1} \in E(G)$. 我们用 $x_{i}, z_{i}, x_{j}, y_{i}, z_{i+1}$ 和 $y_{j}$ 分别替代 $x_{j}, y_{j}, z_{j}, x_{i}, y_{i}$ 和 $z_{i}$.

结合情形 $1-3$, 引理成立.

对于整数 $k$ 和 $s$ 且 $1 \leqslant s \leqslant 4$, 定义函数 $g(k, s)$ 和 $h(s)$ 如下:

\begin{tabular}{ccccc}
\hline$s$ & 1 & 2 & 3 & 4 \\
\hline$g(k, s)$ & $3 k-1$ & $3 k-10$ & $3 k-26$ & $2.5 k-26$ \\
\hline$h(s)$ & 1 & 2 & 4 & 4 \\
\hline
\end{tabular}

如果图 $G$ 的每一个顶点的度在 $k$ 和 $k+s$ 之间, 则称 $G$ 为 $(k, k+s)$-图.

引理 $3^{[19]}$ 设整数 $k$ 和 $s$ 且 $1 \leqslant s \leqslant 4$, 设 $G$ 是 2 -连通的 $(k, k+s)$-图, 其阶为 $n \leqslant g(k, s)$, 则 $G$ 有一个长至少为 $n-h(s)$ 的圈. 进一步, 如果 $\omega(G-A) \leqslant|A|$, 对于所有 $A \subset V(G)$ 且 $|A| \geqslant \frac{1}{2}(n-h(s))$, 则 $G$ 是 Hamilton 图.

引理 4 设 $G$ 是奇数阶的 $(k+1, k+3)$-图, 且设 $|V(G)|=n=2 m+1$ 和 $k \geqslant \frac{n}{3}+8$. 设 $x, y \in V(G)$ 和 $x \neq y, H=G-\{x, y\}$ 是 2-连通的 $(k, k+3)$-图, 且 $H$ 是非 Hamilton 图. 如 果下面条件之一成立:

(a) $G$ 至多有 $\frac{n}{3}$ 个度为 $k+3$ 的顶点且至多有两个度为 $k+1$ 的顶点,

(b) $G$ 至少有 $\frac{2 n}{3}-p$ 个度为 $k+3$ 的顶点且至多有 $q$ 个度为 $k+1$ 的顶点, 这里 $p$ 和 $q$ 是正整数且 $p+q \leqslant 5$, 则存在 $V(H)$ 的一个子集 $A$, 使得 $|A|=m-1$ 且 $\omega(H-A)=m$.

证明 由引理的假设, 有 $|V(H)|=n-2 \leqslant 3 k-26$. 因为 $H$ 是 2-连通的 $(k, k+3)$-图, 且 $H$ 是非 Hamilton 图, 所以由引理 3 , 存在 $V(H)$ 的子集 $A$, 使得 $|A| \geqslant \frac{1}{2}((n-2)-4)=m-\frac{5}{2}$ 且 $\omega(H-A)>|A|$. 也就是 $|A| \geqslant m-2$ 且 $\omega(H-A) \geqslant m-1$. 设 $B=V(H-A)$ 和 $|A|=m-2$, 则 $|B|=m+1$, 因此图 $H-A$ 至多有两条边.

现在假设 $G$ 满足引理的条件 (a). 因为 $G$ 至多有两个度为 $k+1$ 的顶点, 又因为 $H-A$ 至多有两条边且 $|B|=m+1$, 所以, 在 $G$ 中, $B$ 的顶点连接 $A \cup\{x, y\}$ 的顶点的边数至少为 $(k+2)(m+1)-6$, 也就是

$$
e_{G}(B, A \cup\{x, y\}) \geqslant(k+2)(m+1)-6 .
$$

另一方面, 因为 $G$ 至多有 $\frac{n}{3}$ 个度为 $k+3$ 的顶点, 又因为 $\Delta(G) \leqslant k+3$ 以及 $|A \cup\{x, y\}|=m$, 所以, 在 $G$ 中, $A \cup\{x, y\}$ 的顶点连接 $B$ 的顶点的边数至多为 $(k+2) m+\frac{n}{3}$, 也就是

$$
e_{G}(B, A \cup\{x, y\}) \leqslant(k+2) m+\frac{n}{3},
$$

则 $(k+2) m+\frac{n}{3} \geqslant(k+2)(m+1)-6$, 那么, $\frac{n}{3}+4 \geqslant k$. 这与引理的假设矛盾, 因此 $|A|=m-1$ 且 $\omega(H-A)=m$.

再假设 $G$ 满足引理的条件 (b). 在此情形下, 因为图 $H-A$ 至多有两条边且 $|B|=m+1$, 因此, 在 $G$ 中, $B$ 的顶点连接 $A \cup\{x, y\}$ 的顶点的边数至少为

也就是,

$$
(k+3)(m+1)-\left(n-\left(\frac{2 n}{3}-p\right)\right)-q-4,
$$

$$
e_{G}(B, A \cup\{x, y\}) \geqslant(k+3)(m+1)-\left(n-\left(\frac{2 n}{3}-p\right)\right)-q-4
$$




$$
\geqslant(k+3)(m+1)-\frac{n}{3}-p-q-4 .
$$

另一方面, 因为 $|A \cup\{x, y\}|=m$ 和 $\Delta(G)=k+3$, 所以在 $G$ 中, $A \cup\{x, y\}$ 的顶点连接 $B$ 的 顶点的边数至多为 $(k+3) m$, 也就是,

$$
e_{G}(A \cup\{x, y\}, B) \leqslant(k+3) m .
$$

因此

$$
(k+3) m \geqslant(k+3)(m+1)-\frac{n}{3}-p-q-4,
$$

那么 $\frac{n}{3}+1+p+q \geqslant k$, 因为 $k \geqslant \frac{n}{3}+8$, 所以 $p+q \geqslant 7$. 这与引理的假设 (b) 矛盾. 从而 $|A|=m-1$ 且 $\omega(H-A)=m$.

引理 $5^{[14]}$ 设 $G$ 是 $n$ 阶图且 $\delta(G) \geqslant \frac{n}{3}$. 如果 $B \subset V(G)$ 且 $B$ 是 $G$ 的独立集, 使得 $|B|>\frac{n}{3}+1$, 则 $G$ 是 2 -连通的.

下面的结果是引理 5 的一个推论.

推论 1 设 $G$ 是奇数阶的图, $|V(G)|=n$ 且 $\delta(G) \geqslant \frac{n}{3}+10$. 设 $u_{j}, v_{j} \in V(G)$, 使得 $u_{j} \neq v_{j}$ 且 $H_{j}=G-\left\{u_{j}, v_{j}\right\}$, 对于 $j=1,2$. 如果 $V\left(H_{1}\right)$ 的一个子集 $B$ 是 $H_{1}$ 的一个独立 集, 使得

$$
|B|=\frac{n-1}{2}
$$

则 $H_{2}$ 是 2 -连通的.

引理 6 设 $G$ 是一个图, $A$ 和 $B$ 是 $V(G)$ 的两个子集, 使得 $A \cap B=\emptyset$ 和 $|A|=m-1$ 以 及 $|B|=m$. 如果 $X$ 是 $V(G)$ 的一个子集, 使得 $|X|=m-1$ 且 $|A \backslash X| \leqslant l$, 则 $|B \backslash X| \geqslant m-l$.

证明 因为 $|A \backslash X| \leqslant l$, 所以 $|A|-|A \cap X| \leqslant l$, 又因为 $|A|=m-1$, 所以 $|A \cap X| \geqslant$ $|A|-l=m-1-l$. 因为 $|X|=m-1$, 所以 $|B \cap X| \leqslant|X|-|A \cap X| \leqslant(m-1)-(m-1-l)=l$. 又因为 $|B|=m$, 所以 $|B \backslash X|=|B|-|B \cap X| \geqslant m-l$.

下面引理是文献 [12] 的定理 3.1 的证明的直接推论.

引理 7 设 $G$ 是奇数阶的图且 $|V(G)|=n$. 设 $\Delta(G) \leqslant n-2$ 且 $k=n-\delta(G)-2$. 设 存在 $V(G)$ 的一个子集 $\left\{x_{1}, y_{1}, z_{1}, \ldots, x_{k}, y_{k}, z_{k}\right\}$, 使得对于 $1 \leqslant i \leqslant k$, 有 $x_{i} y_{i} \notin E(G)$. 如果 存在 $G$ 的边不相交的匹配 $F_{1}, \ldots, F_{k}$, 使得对于 $1 \leqslant i \leqslant k, F_{i}$ 除顶点 $x_{i}$ 和 $y_{i}$ 及 $z_{i}$ 之外, 饱 和 $G$ 中其他的顶点, 则 $\chi_{T}(G) \leqslant \Delta(G)+2$.

\section{3 主要结果}

定理 1 设 $G$ 奇数阶正则图并且

$$
d(G) \geqslant \frac{2}{3}|V(G)|+\frac{7}{2},
$$

则 $\chi_{T}(G) \leqslant \Delta(G)+2$.

证明 设 $|V(G)|=n=2 m+1$. 如果 $n \leqslant 54$, 则

$$
d(G) \geqslant \frac{2 n}{3}+\frac{7}{2} \geqslant \frac{3 n}{4}-1
$$

1186 
那么由文献 [12] 中定理 3.1, 此时结论成立. 因此, 我们假设 $n \geqslant 55$. 如果 $d(G) \geqslant \frac{5 n}{6}-3$, 由 $n \geqslant 55$, 则 $d(G) \geqslant \frac{3 n}{4}-1$, 因此由文献 [12] 中定理 3.1, 此时结论成立. 我们还假设 $d(G)<\frac{5 n}{6}-3$. 如果 $d(G) \geqslant n-2$, 则这种情形结论成立 ${ }^{[13]}$. 我们还假设 $d(G) \leqslant n-3$.

设 $k=n-d(G)-2$. 由定理和引理 2 的假设, 存在 $V(G)$ 的一个子集 $\left\{x_{1}, y_{1}, z_{1}, \ldots, x_{k}, y_{k}\right.$, $\left.z_{k}\right\}$, 使得对于 $1 \leqslant i \leqslant k$, 有 $x_{i} y_{i} \notin E(G)$ 且 $y_{i} z_{i} \in E(G)$.

设 $r=\left\lceil\frac{n}{6}\right\rceil+1$. 因为 $d(G)<\frac{5 n}{6}-3$, 所以 $k=n-d(G)-2>n-\left(\frac{5 n}{6}-3\right)-2=\frac{n}{6}+1$, 那么 $k \geqslant r$. 设 $V_{1}=\left\{x_{1}, y_{1}, \ldots, x_{r}, y_{r}, \ldots, x_{k}, y_{k}\right\}$ 和 $E_{1}=\left\{y_{1} z_{1}, \ldots, y_{r} z_{r}\right\}$.

引理 8 存在 $G-E_{1}$ 的边不相交的匹配 $F_{1}, \ldots, F_{r}$, 使得对于 $1 \leqslant i \leqslant r, F_{i}$ 不饱和 $x_{i}, y_{i}$ 和 $z_{i}$, 而饱和 $G$ 的所有其他的顶点.

证明 假设对于 $F_{1}, \ldots, F_{i-1}, i \leqslant r$, 引理成立. 设 $G_{i}=G-\left(E_{1} \cup F_{1} \cup \cdots \cup F_{i-1}\right)$ 和 $H_{i}=G_{i}-\left\{x_{i}, y_{i}\right\}$. 由 $(2)$ 式,

$$
\begin{aligned}
\delta\left(H_{i}\right) & \geqslant d(G)-i-2 \geqslant d(G)-r-2 \geqslant \frac{2 n}{3}+\frac{7}{2}-\left(\left\lceil\frac{n}{6}\right\rceil+1\right)-2 \\
& =\frac{2 n}{3}+\frac{1}{2}-\left\lceil\frac{n}{6}\right\rceil \geqslant \frac{2 n}{3}+\frac{1}{2}-\left(\frac{n}{6}+1\right)=\frac{n-1}{2} \geqslant \frac{1}{2}\left|V\left(H_{i}\right)\right|,
\end{aligned}
$$

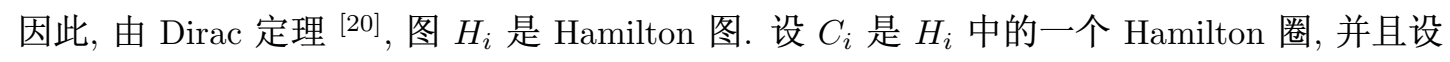
$F_{j}$ 是 $H_{i}$ 中最大的匹配, 使得 $F_{j} \subset E\left(C_{i}\right)$ 且 $z_{i}$ 不与 $F_{j}$ 中的边相关联. 令 $F_{i}=F_{j}$, 则引理 成立.

引理 9 对于 $k \leqslant\left\lfloor\frac{2 n}{9}\right\rfloor+1$, 存在 $G-E_{1}$ 的边不相交的匹配 $F_{1}, \ldots, F_{k}$, 使得对于 $1 \leqslant i \leqslant k, F_{i}$ 不饱和 $x_{i}, y_{i}$ 和 $z_{i}$, 而饱和 $G$ 的所有其他的顶点.

证明 由引理 8 , 对于 $k \leqslant r$ 时引理成立. 假设对于 $r \leqslant i-1 \leqslant k-1$ 时引理成立. 设 $G_{i}=$ $G-\left(E_{1} \cup F_{1} \cup \cdots \cup F_{i-1}\right)$ 和 $H_{i}=G_{i}-\left\{x_{i}, y_{i}\right\}$. 设 $V_{2}=\left\{x_{1}, \ldots, x_{r}, x_{r+1}, y_{r+1}, z_{r+1}, \ldots, x_{i-1}\right.$, $\left.y_{i-1}, z_{i-1}\right\}$, 因为 $G$ 是正则图 (注意 $E_{1}$ 的选择), 所以, 对于 $u \in V_{2}$, 有 $d_{G_{i}}(u)=\Delta\left(G_{i}\right)$; 对于 $u \in V\left(G_{i}\right) \backslash V_{2}$, 有 $d_{G_{i}}(u)=\Delta\left(G_{i}\right)-1=\delta\left(G_{i}\right)$. 因此, $\Delta\left(G_{i}\right)=\delta\left(G_{i}\right)+1$ 且

$$
\Delta\left(G_{i}\right)-3 \leqslant \delta\left(H_{i}\right) \leqslant \Delta\left(H_{i}\right) \leqslant \Delta\left(G_{i}\right) .
$$

设 $\Delta=\Delta\left(G_{i}\right)$, 则 $G_{i}$ 是一个 $(\Delta-1, \Delta)$-图并且 $H_{i}$ 是一个 $(\Delta-3, \Delta)$-图. 由 (1) 和 (2) 式 (注意 $E_{1}$ 的选择),

$$
\begin{aligned}
\delta\left(H_{i}\right) & \geqslant d(G)-(i-1)-2 \geqslant d(G)-(k-1)-2 \text { (因为 } i \leqslant k) \\
& =d(G)-k-1 \geqslant\left(\frac{2 n}{3}+\frac{7}{2}\right)-\left(\frac{n}{3}-\frac{11}{2}\right)-1=\frac{n}{3}+8 .
\end{aligned}
$$

类似地,

$$
\delta\left(G_{i}\right) \geqslant \frac{n}{3}+10
$$

因此 $\Delta\left(G_{i}\right)=\delta\left(G_{i}\right)+1 \geqslant \frac{n}{3}+11$, 所以 $\Delta-3 \geqslant \frac{n}{3}+8$.

情形 $1 \quad H_{i}$ 是 2-连通的.

若 $H_{i}$ 是 Hamilton 图. 类似于引理 8 , 引理成立. 现在假设 $H_{i}$ 是非 Hamilton 图. 因为

$$
r=\left\lceil\frac{n}{6}\right\rceil+1 \text { 和 } i-1 \leqslant k-1 \leqslant\left\lfloor\frac{2 n}{9}\right\rfloor,
$$


所以

$$
\begin{aligned}
\left|V_{2}\right| & =r+3(i-1-r)=3(i-1)-2 r \\
& \leqslant 3\left\lfloor\frac{2 n}{9}\right\rfloor-2\left(\left\lceil\frac{n}{6}\right\rceil+1\right) \\
& \leqslant 3\left(\frac{2 n}{9}\right)-2\left(\frac{n}{6}+1\right)<\frac{n}{3},
\end{aligned}
$$

因此, $G_{i}$ 至多有 $\frac{n}{3}$ 个度为 $\Delta$ 的顶点. 因为 $H_{i}$ 还是 $2-$ 连通的, 所以 $G_{i}$ 和 $H_{i}$ 满足引理 4 的假 设, 从而存在 $V\left(H_{i}\right)$ 的一个子集 $A$, 使得 $|A|=m-1$ 并且 $\omega\left(H_{i}-A\right)=m$. 设 $B=V\left(H_{i}-A\right)$, 则 $B$ 是 $H_{i}$ 的一个独立集并且 $|B|=m$.

断言 1 在 $G_{i}$ 中, 两个顶点 $x_{i}$ 和 $y_{i}$ 都至少与 $B$ 中的 5 个顶点相邻.

证明 假设结论不成立. 不失一般性, 假设在 $G_{i}$ 中, $y_{i}$ 至多与 $B$ 中 4 个顶点相邻, 则在 $G_{i}$ 中, $y_{i}$ 至少与 $A \cup\left\{x_{i}\right\}$ 中的 $\delta\left(G_{i}\right)-4$ 个顶点相邻. 因为 $B$ 是 $H_{i}$ 的独立集且 $\left|B \cup\left\{y_{i}\right\}\right|=$ $m+1$, 所以在 $G_{i}$ 中, 连接 $B \cup\left\{y_{i}\right\}$ 与 $A \cup\left\{x_{i}\right\}$ 的顶点的边数至少是 $\delta\left(G_{i}\right)(m+1)-8$, 即

$$
e_{G_{i}}\left(B \cup\left\{y_{i}\right\}, A \cup\left\{x_{i}\right\}\right) \geqslant \delta\left(G_{i}\right)(m+1)-8 .
$$

另一方面, 因为 $G_{i}$ 有至多 $\frac{n}{3}$ 个度为 $\Delta$ 的顶点, 并且因为 $\Delta\left(G_{i}\right) \leqslant \delta\left(G_{i}\right)+1$ 和 $\left|A \cup\left\{x_{i}\right\}\right|=m$, 所以在 $G_{i}$ 中, 连接 $A \cup\left\{x_{i}\right\}$ 与 $B \cup\left\{y_{i}\right\}$ 的顶点的边数至多是 $\delta\left(G_{i}\right) m+\frac{n}{3}$, 也就是

$$
e_{G_{i}}\left(A \cup\left\{x_{i}\right\}, B \cup\left\{y_{i}\right\}\right) \leqslant \delta\left(G_{i}\right) m+\frac{n}{3},
$$

因此 $\delta\left(G_{i}\right)(m+1)-8 \leqslant \delta\left(G_{i}\right) m+\frac{n}{3}$, 所以 $\frac{n}{3}+8 \geqslant \delta\left(G_{i}\right)$, 这与 $(5)$ 式矛盾, 故断言成立.

类似地我们有如下结果:

断言 2 如果 $\left|E\left(H_{i}[A]\right)\right| \leqslant 1$, 则在 $G_{i}$ 中, $\left\{x_{i}, y_{i}\right\}$ 中顶点之一与 $A$ 中的至少两个顶点 相邻.

断言 3 在 $G_{i}$ 中, $A$ 的每一个顶点至少与 $B$ 的 5 个顶点相邻.

由 (1) 式, $\left|V(G) \backslash V_{1}\right|=n-2 k \geqslant n-2\left(\frac{n}{3}-\frac{11}{2}\right)=\frac{n}{3}+11>k$. 设 $V(G) \backslash V_{1}=$ $\left\{z_{1}, \ldots, z_{k}, \ldots, z_{l}\right\}$, 则 $l>k$.

情形 1.1 若 $B \cap\left\{z_{i}, \ldots, z_{k}, \ldots, z_{l}\right\} \neq \emptyset$.

不失一般性, 假设 $z_{i} \in B$. 如果 $A$ 是 $H_{i}$ 的一个独立集, 则由断言 2 , 可以假设在 $G_{i}$ 中, $y_{i}$ 至少与 $A$ 的两个顶点相邻. 设 $H_{i}^{\prime}=G_{i}-\left\{x_{i}, z_{i}\right\}$, 和 (3) 式类似, 有

$$
\Delta\left(G_{i}\right)-3 \leqslant \delta\left(H_{i}^{\prime}\right) \leqslant \Delta\left(H_{i}^{\prime}\right) \leqslant \Delta\left(G_{i}\right)
$$

和

$$
\delta\left(H_{i}^{\prime}\right) \geqslant \frac{n}{3}+8 .
$$

因此, $H_{i}^{\prime}$ 是一个 $(\Delta-3, \Delta)$-图. 因为 $B$ 是 $H_{i}$ 中的一个独立集, 所以, 由推论 1 , 得 $H_{i}^{\prime}$ 是 2 -连通的. 我们将证明 $H_{i}^{\prime}$ 是 Hamilton 图. 假设结论不成立. 则 $G_{i}$ 和 $H_{i}^{\prime}$ 都满足引理 4 的假 设, 从而存在 $V\left(H_{i}^{\prime}\right)$ 的一个子集 $A^{\prime}$, 使得 $\left|A^{\prime}\right|=m-1$ 且 $\omega\left(H_{i}^{\prime}-A^{\prime}\right)=m$. 设 $B^{\prime}=V\left(H_{i}^{\prime}-A^{\prime}\right)$, 则 $B^{\prime}$ 是 $H_{i}^{\prime}$ 的一个独立集, 并且 $\left|B^{\prime}\right|=m$.

若 $B \cap B^{\prime}=\emptyset$, 因为 $|A|=m-1$ 和 $|B|=m=\left|B^{\prime}\right|$ 以及 $B \cap A=\emptyset$, 所以 $B^{\prime}=A \cup\left\{y_{i}\right\}$, 则 $A$ 是 $H_{i}^{\prime}$ 的一个独立集. 因此, $A$ 也是 $H_{i}$ 的一个独立集. 由 $y_{i}$ 的选择 (注意 $z_{i} \notin A$ ), 在 
$H_{i}^{\prime}$ 中, 顶点 $y_{i}$ 与 $A$ 中至少两个顶点相邻. 这与 $B^{\prime}$ 是 $H_{i}^{\prime}$ 的独立集相矛盾, 因此 $B \cap B^{\prime} \neq \emptyset$. 因为 $B$ 是 $H_{i}$ 的一个独立集, 所以存在一个顶点 $u \in B \cap B^{\prime}$, 使得在 $H_{i}$ 中, $u$ 至少与 $A$ 的 $\delta\left(H_{i}\right)$ 个顶点相邻. 因为 $z_{i} \notin A$, 所以在 $H_{i}-\left\{z_{i}\right\}$ 中, $u$ 至少与 $A$ 的 $\delta\left(H_{i}\right)$ 个顶点相邻, 因 为 $H_{i}-\left\{z_{i}\right\}$ 是 $H_{i}^{\prime}$ 的一个子图和 $B^{\prime}$ 是 $H_{i}^{\prime}$ 的一个独立集, 所以在 $H_{i}^{\prime}$ 中, $u$ 至少与 $A \cap A^{\prime}$ 的 $\delta\left(H_{i}\right)$ 个顶点相邻, 因此

$$
\left|A \cap A^{\prime}\right| \geqslant \delta\left(H_{i}\right) \geqslant \frac{n}{3}+8 .
$$

情形 1.1.1 若 $\left|A \backslash A^{\prime}\right|=0$. 因为 $|A|=m-1=\left|A^{\prime}\right|$, 所以 $A=A^{\prime}$. 又因为 $z_{i} \in B$, 由 $B$ 和 $B^{\prime}$ 的定义, 有 $B^{\prime}=\left(B \backslash\left\{z_{i}\right\}\right) \cup\left\{y_{i}\right\}$. 由断言 1 , 在 $G_{i}$ 中, $y_{i}$ 至少与 $B \backslash\left\{z_{i}\right\}$ 的 4 个顶 点相邻, 则在 $H_{i}^{\prime}$ 中, $y_{i}$ 至少与 $B \backslash\left\{z_{i}\right\}$ 的 4 个顶点相邻, 因此, $B^{\prime}$ 不是 $H_{i}^{\prime}$ 的一个独立集, 矛盾.

情形 1.1.2 若 $1 \leqslant\left|A \backslash A^{\prime}\right| \leqslant 3$. 由引理 $6,\left|B \backslash A^{\prime}\right| \geqslant m-3$. 由断言 3, 存在一个顶点 $v \in A \backslash A^{\prime} \subset B^{\prime}$, 使得在 $G_{i}$ 中, $v$ 至少与 $B \backslash\left(A^{\prime} \cup\left\{z_{i}\right\}\right)$ 的一个顶点相邻, 则在 $H_{i}^{\prime}$ 中, $v$ 至 少与 $B \backslash\left(A^{\prime} \cup\left\{z_{i}\right\}\right)$ 的一个顶点相邻. 因为 $B \backslash\left(A^{\prime} \cup\left\{z_{i}\right\}\right) \in B^{\prime}$, 所以 $B^{\prime}$ 不是 $H_{i}^{\prime}$ 的独立集, 矛盾.

情形 1.1.3 若 $\left|A \backslash A^{\prime}\right| \geqslant 4$. 设 $p=\left|A \backslash A^{\prime}\right|$, 因为 $B$ 是 $H_{i}$ 的一个独立集, 由 $d_{G_{i}}\left(x_{i}\right)=$ $\delta\left(G_{i}\right)=d_{G_{i}}\left(y_{i}\right)$ 和 $|B|=m$, 因此, 在 $H_{i}$ 中, $B$ 的顶点连接 $A$ 的顶点的边数至少为 $\delta\left(G_{i}\right) m-$ $2 \delta\left(G_{i}\right)$. 因为 $G_{i}$ 有至多 $\frac{n}{3}$ 个度为 $\Delta$ 的顶点, 所以 $H_{i}$ 有至多 $\frac{n}{3}$ 个度为 $\Delta\left(G_{i}\right)$ 的顶点. 因为 $\Delta\left(H_{i}\right) \leqslant \Delta\left(G_{i}\right) \leqslant \delta\left(G_{i}\right)+1$ 和 $|A|=m-1$, 所以

$$
\begin{aligned}
\left|E\left(H_{i}[A]\right)\right| & \leqslant \frac{\left(\delta\left(G_{i}\right)(m-1)+\frac{n}{3}\right)-\left(\delta\left(G_{i}\right) m-2 \delta\left(G_{i}\right)\right)}{2} \\
& =\frac{1}{2} \delta\left(G_{i}\right)+\frac{n}{6} \\
& \left.\leqslant \frac{1}{2} \delta\left(H_{i}\right)+1+\frac{n}{6} \quad \text { (因为 } \delta\left(G_{i}\right) \leqslant \delta\left(H_{i}\right)+2\right) \\
& =\delta\left(H_{i}\right)-\frac{1}{2} \delta\left(H_{i}\right)+1+\frac{n}{6} \\
& \leqslant \delta\left(H_{i}\right)-\frac{1}{2}\left(\frac{n}{3}+8\right)+1+\frac{n}{6} \text { (由 (4) 式) } \\
& \leqslant \delta\left(H_{i}\right)-3,
\end{aligned}
$$

因此 $e_{H_{i}}\left(A \backslash A^{\prime}, B\right) \geqslant p \delta\left(H_{i}\right)-2\left(\delta\left(H_{i}\right)-3\right)$, 从而存在一个顶点 $v \in A \backslash A^{\prime} \subset B^{\prime}$, 使得

$$
\begin{aligned}
e_{H_{i}}(\{v\}, B) & \geqslant \frac{p \delta\left(H_{i}\right)-2\left(\delta\left(H_{i}\right)-3\right)}{p} \\
& \left.=\frac{(p-2) \delta\left(H_{i}\right)}{p}+\frac{6}{p} \geqslant \frac{2}{4} \delta\left(H_{i}\right) \quad \text { (因为 } p \geqslant 4\right) \\
& \geqslant \frac{1}{2}\left(\frac{n}{3}+8\right) \text { (由 }(4) \text { 式) } \\
& =\frac{n}{6}+4 .
\end{aligned}
$$


因为 $\left|A^{\prime}\right|=\frac{n-1}{2}-1$, 由 (8) 式, 所以

$$
\left|B \cap A^{\prime}\right| \leqslant\left|A^{\prime}\right|-\left|A \cap A^{\prime}\right| \leqslant \frac{n-1}{2}-1-\left(\frac{n}{3}+8\right)=\frac{n}{6}-\frac{19}{2} .
$$

由 (10) 和 (11) 式,

$$
\begin{aligned}
e_{H_{i}^{\prime}}\left(\{v\}, B \backslash\left(A^{\prime} \cup\left\{z_{i}\right\}\right)\right) & =e_{H_{i}}\left(\{v\}, B \backslash\left(A^{\prime} \cup\left\{z_{i}\right\}\right)\right) \\
& =e_{H_{i}}\left(\{v\}, B \backslash\left(\left(B \cap A^{\prime}\right) \cup\left\{z_{i}\right\}\right)\right) \\
& \geqslant\left(\frac{n}{6}+4\right)-\left(\frac{n}{6}-\frac{19}{2}\right)-1>12 .
\end{aligned}
$$

因为 $v \in B^{\prime}$ 和 $B \backslash\left(A^{\prime} \cup\left\{z_{i}\right\}\right) \subset B^{\prime}$, 所以 $B^{\prime}$ 不是 $H_{i}^{\prime}$ 的独立集, 矛盾.

因此, $H_{i}^{\prime}$ 是 Hamilton 图. 设 $C_{i}^{\prime}$ 是 $H_{i}^{\prime}$ 中的一个 Hamilton 圈并且设 $F_{i}$ 是 $H_{i}^{\prime}$ 中的最大 匹配, 使得 $F_{i} \subset E\left(C_{i}^{\prime}\right)$ 且 $y_{i}$ 不与 $F_{i}$ 中的边相关联.

情形 1.2 若 $B \cap\left\{z_{i}, \ldots, z_{k}, \ldots, z_{l}\right\}=\emptyset$, 但 $B \cap\left\{x_{i+1}, y_{i+1}, \ldots, x_{k}, y_{k}\right\} \neq \emptyset$.

则 $\left\{z_{i}, \ldots, z_{k}, \ldots, z_{l}\right\} \subset A$. 因为 $B \cap\left\{x_{i+1}, y_{i+1}, \ldots, x_{k}, y_{k}\right\} \neq \emptyset$, 因此, 不失一般性, 假 设 $x_{i+1} \in B$. 因为 $i \leqslant k$, 由 $(1)$ 式, $3(i-1) \leqslant 3 k \leqslant n-\frac{33}{2}$, 则 $\left|\left\{z_{i}, \ldots, z_{l}\right\}\right| \geqslant 2$. 如果在 $G_{i}$ 中, $x_{i}$ 和 $y_{i}$ 都不与 $A$ 的至少两个顶点相邻, 由断言 $2,\left|E\left(H_{i}[A]\right)\right| \geqslant 2$, 则存在 $\left\{z_{i}, \ldots, z_{k}, \ldots, z_{l}\right\}$ 的 1 个顶点记为 $z_{j}$, 使得 $\left|E\left(H_{i}\left[A \backslash\left\{z_{j}\right\}\right]\right)\right| \geqslant 1$. 不失一般性, 假设 $z_{j}=z_{i+1}$. 我们分别用 $x_{i}^{\prime}$, $y_{i}^{\prime}$ 和 $z_{i}^{\prime}$ 表示 $x_{i+1}, z_{i+1}$ 和 $y_{i+1}$, 则 $x_{i}^{\prime} \in B$ 和 $y_{i}^{\prime} \in A$. 设 $H_{i}^{\prime}=G_{i}-\left\{x_{i}^{\prime}, y_{i}^{\prime}\right\}$. 类似地, 有 (6) 和 (7) 式, $H_{i}^{\prime}$ 是 $(\Delta-3, \Delta)$-图, 且 $H_{i}^{\prime}$ 是 2-连通的. 假设 $H_{i}^{\prime}$ 是非 Hamilton 图, 则 $G_{i}$ 和 $H_{i}^{\prime}$ 满足引理 4 的假设, 从而存在 $V\left(H_{i}^{\prime}\right)$ 的子集 $A^{\prime}$, 使得 $\left|A^{\prime}\right|=m-1$ 和 $\omega\left(H_{i}^{\prime}-A^{\prime}\right)=m$. 设 $B^{\prime}=V\left(H_{i}^{\prime}-A^{\prime}\right)$, 则 $B^{\prime}$ 是 $H_{i}^{\prime}$ 的一个独立集并且 $\left|B^{\prime}\right|=m$.

若 $B \cap B^{\prime}=\emptyset$. 因为 $|B|=m=\left|B^{\prime}\right|$ 和 $y_{i}^{\prime} \in A$ 以及 $A \cap B=\emptyset$, 从而有 $B^{\prime}=$ $\left\{x_{i}, y_{i}\right\} \cup\left(A \backslash\left\{y_{i}^{\prime}\right\}\right)$. 因此, 由 $y_{i}^{\prime}$ 的选择, 或者 $\left|E\left(H_{i}\left[A \backslash\left\{y_{i}^{\prime}\right\}\right]\right)\right| \geqslant 1$, 或者在 $G_{i}$ 中, $\left\{x_{i}, y_{i}\right\}$ 中之一至少与 $A \backslash\left\{y_{i}^{\prime}\right\}$ 的一个顶点相邻, 也就是, 或者 $\left|E\left(H_{i}^{\prime}\left[A \backslash\left\{y_{i}^{\prime}\right\}\right]\right)\right| \geqslant 1$, 或者在 $H_{i}^{\prime}$ 中, $\left\{x_{i}, y_{i}\right\}$ 中之一至少与 $A \backslash\left\{y_{i}^{\prime}\right\}$ 的 1 个顶点相邻, 那么 $B^{\prime}$ 不是 $H_{i}^{\prime}$ 的一个独立集, 矛盾, 因此 $B \cap B^{\prime} \neq \emptyset$. 因为 $x_{i}^{\prime} \in B$ 和 $y_{i}^{\prime} \in A$, 如同 (8) 式, 有

$$
\left|A \cap A^{\prime}\right| \geqslant \delta\left(H_{i}\right)-1 \geqslant \frac{n}{3}+7 \text {. }
$$

情形 1.2.1 若 $\left|A \backslash A^{\prime}\right|=1$, 则 $A \backslash A^{\prime}=\left\{y_{i}^{\prime}\right\}$. 因为 $|A|=m-1=\left|A^{\prime}\right|$, 由 $\mathrm{B}$ 和 $B^{\prime}$ 的定 义, 有 $x_{i} \in B^{\prime}$ 或者 $y_{i} \in B^{\prime}$. 不失一般性, 假设 $x_{i} \in B^{\prime}$. 由引理 $6,\left|B \backslash A^{\prime}\right| \geqslant m-1$. 由断言 1 , 在 $G_{i}$ 中, $x_{i}$ 至少与 $B \backslash\left(A^{\prime} \cup\left\{x_{i}^{\prime}\right\}\right)$ 的 3 个顶点相邻, 则在 $H_{i}^{\prime}$ 中, $x_{i}$ 至少与 $B \backslash\left(A^{\prime} \cup\left\{x_{i}^{\prime}\right\}\right)$ 的 3 个顶点相邻. 因为 $B \backslash\left(A^{\prime} \cup\left\{x_{i}^{\prime}\right\}\right) \subset B^{\prime}$, 所以 $B^{\prime}$ 不是 $H_{i}^{\prime}$ 的一个独立集, 矛盾.

情形 1.2.2 若 $2 \leqslant\left|A \backslash A^{\prime}\right| \leqslant 3$. 由引理 $6,\left|B \backslash A^{\prime}\right| \geqslant m-3$, 则 $\left|B \backslash\left(A^{\prime} \cup\left\{x_{i}^{\prime}\right\}\right)\right| \geqslant m-4$. 因为 $\left|A \backslash A^{\prime}\right| \geqslant 2$, 由断言 3 , 存在顶点 $u \in A \backslash\left(A^{\prime} \cup\left\{y_{i}^{\prime}\right\}\right) \subset B^{\prime}$, 使得在 $G_{i}$ 中, $u$ 至少与 $B \backslash\left(A^{\prime} \cup\left\{x_{i}^{\prime}\right\}\right)$ 的 1 个顶点相邻, 那么, 在 $H_{i}^{\prime}$ 中, $u$ 至少与 $B \backslash\left(A^{\prime} \cup\left\{x_{i}^{\prime}\right\}\right)$ 的 1 个顶点相邻. 因为 $B \backslash\left(A^{\prime} \cup\left\{x_{i}^{\prime}\right\}\right) \subset B^{\prime}$, 所以 $B^{\prime}$ 不是 $H_{i}^{\prime}$ 的一个独立集, 矛盾.

情形 1.2.3 若 $4 \leqslant\left|A \backslash A^{\prime}\right| \leqslant 5$. 由引理 $6,\left|B \backslash A^{\prime}\right| \geqslant m-5$, 则 $\left|B \backslash\left(A^{\prime} \cup\left\{x_{i}^{\prime}\right\}\right)\right| \geqslant m-6$. 因为 $\left|A \backslash A^{\prime}\right| \geqslant 4$, 由 (9) 式, 存在 $u, v \in A \backslash\left(A^{\prime} \cup\left\{y_{i}^{\prime}\right\}\right)$, 使得

$$
e_{H_{i}}(\{u, v\}, B) \geqslant 2 \delta\left(H_{i}\right)-\left(\delta\left(H_{i}\right)-3\right)-1
$$




$$
=\delta\left(H_{i}\right)+2 \geqslant \frac{n}{3}+10 \geqslant 28 \text { (因为 } n>55 \text { ). }
$$

因此, $e_{H_{i}}\left(\{u, v\}, B \backslash\left(A^{\prime} \cup\left\{x_{i}^{\prime}\right\}\right)\right) \geqslant 28-12=16$, 从而,

$$
e_{H_{i}-\left\{x_{i}^{\prime}, y_{i}^{\prime}\right\}}\left(\{u, v\}, B \backslash\left(A^{\prime} \cup\left\{x_{i}^{\prime}\right\}\right)\right) \geqslant 16 .
$$

因为 $H_{i}-\left\{x_{i}^{\prime}, y_{i}^{\prime}\right\}$ 是 $H_{i}^{\prime}$ 的子图, 所以

$$
e_{H_{i}^{\prime}}\left(\{u, v\}, B \backslash\left(A^{\prime} \cup\left\{x_{i}^{\prime}\right\}\right)\right) \geqslant 16 .
$$

因为 $u, v \in A \backslash\left(A^{\prime} \cup\left\{y_{i}^{\prime}\right\}\right) \subset B^{\prime}$ 和 $B \backslash\left(A^{\prime} \cup\left\{x_{i}^{\prime}\right\}\right) \subset B^{\prime}$, 所以 $B^{\prime}$ 不是 $H_{i}^{\prime}$ 的一个独立集, 矛 盾.

情形 1.2.4 若 $\left|A \backslash A^{\prime}\right| \geqslant 6$. 设 $p=\left|A \backslash A^{\prime}\right|$. 因为 $\left|A \backslash\left(A^{\prime} \cup\left\{y_{i}^{\prime}\right\}\right)\right|=p-1$, 由 (9) 式, 所 以 $e_{H_{i}}\left(A \backslash\left(A^{\prime} \cup\left\{y_{i}^{\prime}\right\}\right), B\right) \geqslant(p-1) \delta\left(H_{i}\right)-2\left(\delta\left(H_{i}\right)-3\right)$, 从而存在顶点 $v \in A \backslash\left(A^{\prime} \cup\left\{y_{i}^{\prime}\right\}\right) \subset B^{\prime}$, 使得

$$
\begin{aligned}
e_{H_{i}}(\{v\}, B) & \geqslant \frac{(p-1) \delta\left(H_{i}\right)-2\left(\delta\left(H_{i}\right)-3\right)}{p} \\
& =\frac{(p-3) \delta\left(H_{i}\right)}{p}+\frac{6}{p} \geqslant \frac{3}{6} \delta\left(H_{i}\right) \quad(\text { 因为 } p \geqslant 6, \text { 如同 }(10) \text { 式) } \\
& \geqslant \frac{n}{6}+4 .
\end{aligned}
$$

因为

$$
\left|A^{\prime}\right|=\frac{n-1}{2}-1
$$

由 (12) 式, 所以

$$
\left|B \cap A^{\prime}\right| \leqslant\left|A^{\prime}\right|-\left|A \cap A^{\prime}\right| \leqslant \frac{n-1}{2}-1-\left(\frac{n}{3}+7\right)=\frac{n}{6}-\frac{17}{2},
$$

从而,

$$
\begin{aligned}
e_{H_{i}^{\prime}}\left(\{v\}, B \backslash\left(A^{\prime} \cup\left\{x_{i}^{\prime}\right\}\right)\right) & =e_{H_{i}}\left(\{v\}, B \backslash\left(A^{\prime} \cup\left\{x_{i}^{\prime}\right\}\right)\right) \\
& =e_{H_{i}}\left(\{v\}, B \backslash\left(\left(B \cap A^{\prime}\right) \cup\left\{x_{i}^{\prime}\right\}\right)\right) \\
& \geqslant\left(\frac{n}{6}+4\right)-\left(\frac{n}{6}-\frac{17}{2}\right)-1 \geqslant 11 .
\end{aligned}
$$

因为 $v \in B^{\prime}$ 和 $B \backslash\left(A^{\prime} \cup\left\{x_{i}^{\prime}\right\}\right) \subset B^{\prime}$, 所以 $B^{\prime}$ 不是 $H_{i}^{\prime}$ 的一个独立集, 矛盾.

因此, $H_{i}^{\prime}$ 是 Hamilton 图. 设 $C_{i}^{\prime}$ 是 $H_{i}^{\prime}$ 中的一个 Hamilton 圈并且设 $F_{i}$ 是 $H_{i}^{\prime}$ 中的最大 匹配, 使得 $F_{i} \subset E\left(C_{i}^{\prime}\right)$ 且 $z_{i}^{\prime}$ 不与 $F_{i}$ 中的边相关联. 分别用 $x_{i}^{\prime}, z_{i}^{\prime}$ 和 $y_{i}^{\prime}$ 替代 $x_{i}, y_{i}$ 和 $z_{i}$.

情形 1.3 若 $B \cap\left\{x_{i+1}, \ldots, x_{k}, y_{i+1}, \ldots, y_{k}, z_{i}, \ldots, z_{k}, \ldots, z_{l}\right\}=\emptyset$.

设 $V_{3}=\left\{x_{i+1}, \ldots, x_{k}, y_{i+1}, \ldots, y_{k}, z_{i}, \ldots, z_{k}, \ldots, z_{l}\right\}$, 则 $V_{3} \subset A$. 因为 $i \leqslant k \leqslant\left\lfloor\frac{2 n}{9}\right\rfloor+1$, 所以

$$
\begin{aligned}
\left|V_{3}\right| & =|V(G)|-3 i+1 \geqslant n-3\left(\left\lfloor\frac{2 n}{9}\right\rfloor+1\right)+1 \\
& =n-3\left\lfloor\frac{2 n}{9}\right\rfloor-2 \geqslant n-3\left(\frac{2 n}{9}\right)-2=\frac{n}{3}-2 .
\end{aligned}
$$


因为 $|A|=\frac{n-1}{2}-1$, 所以 $\left|A \backslash V_{3}\right|=|A|-\left|V_{3}\right| \leqslant\left(\frac{n-1}{2}-1\right)-\left(\frac{n}{3}-2\right)=\frac{n}{6}+\frac{1}{2}$. 设 $r_{1}=\left\lfloor\frac{n}{6}+\frac{1}{2}\right\rfloor$. 则 $\left\{F_{1}, \ldots, F_{i-1}\right\}$ 包含至多 $r_{1}$ 个匹配, 记为 $F_{1}, \ldots, F_{r_{1}}$, 使得 $A \subset V_{3} \cup\left\{x_{1}, y_{1}, z_{1}, \ldots, x_{r_{1}}\right.$, $\left.y_{r_{1}}, z_{r_{1}}\right\}$, 因此

$$
\left\{x_{r_{1}+1}, y_{r_{1}+1}, z_{r_{1}+1}, \ldots, x_{i-1}, y_{i-1}, z_{i-1}\right\} \subset B
$$

由 (2) 式,

$$
\delta(G[B]) \geqslant d(G)-|B| \geqslant \frac{2 n}{3}+\frac{7}{2}-m=\frac{2 n}{3}+\frac{7}{2}-\frac{n-1}{2}=\frac{n}{6}+4 .
$$

因为 $|B|=\frac{n-1}{2}$, 所以

且

$$
|E(G[B])| \geqslant \frac{1}{2} \delta(G[B])|B| \geqslant \frac{1}{2}\left(\frac{n}{6}+4\right) \frac{n-1}{2}
$$

$$
\left|E(G[B]) \cap\left(\bigcup_{j=1}^{r_{1}} F_{j}\right)\right| \leqslant \frac{1}{2} r_{1}|B| \leqslant \frac{1}{2}\left(\frac{n}{6}+\frac{1}{2}\right) \frac{n-1}{2} .
$$

我们有 $\left|E(G[B]) \cap E_{1}\right| \leqslant \frac{1}{2}|B| \leqslant \frac{1}{2}\left(\frac{n-1}{2}\right)$. 因此, 由 (13) 和 (14) 式, 得

因为 $r_{1}<r<i \leqslant k$,

$$
\begin{aligned}
\left|E(G[B]) \backslash\left(\bigcup_{j=1}^{r_{1}} F_{j} \cup E_{1}\right)\right| & \geqslant|E(G[B])|-\left|E(G[B]) \cap\left(\bigcup_{j=1}^{r_{1}} F_{j}\right)\right|-\left|E(G[B]) \cap E_{1}\right| \\
& \geqslant \frac{1}{2}\left(\frac{n}{6}+4\right) \frac{n-1}{2}-\frac{1}{2}\left(\frac{n}{6}+\frac{1}{2}\right) \frac{n-1}{2}-\frac{1}{2}\left(\frac{n-1}{2}\right) \\
& =\frac{5}{8}(n-1) .
\end{aligned}
$$

$$
\begin{aligned}
\frac{\left|E(G[B]) \backslash\left(\bigcup_{j=1}^{r_{1}} F_{j} \cup E_{1}\right)\right|}{i-1-r_{1}} & \geqslant \frac{\frac{5(n-1)}{8}}{k-1-r_{1}} \geqslant \frac{\frac{5(n-1)}{8}}{\left(\frac{n}{3}-\frac{11}{2}\right)-1-\left\lfloor\frac{n}{6}+\frac{1}{2}\right\rfloor} \text { (由 (1) 式) } \\
& \geqslant \frac{\frac{5(n-1)}{8}}{\left(\frac{n}{3}-\frac{11}{2}\right)-1-\left(\frac{n}{6}+\frac{1}{2}\right)+1} \\
& \geqslant \frac{5(n-1)}{\frac{n}{6}-6}=\frac{15(n-1)}{4(n-36)} \geqslant 3 .
\end{aligned}
$$

因为 $\mathrm{B}$ 是 $H_{i}$ 的一个独立集, 所以, $E(G[B]) \backslash\left(\bigcup_{j=1}^{r_{1}} F_{j} \cup E_{1}\right) \subset \bigcup_{p=r_{1}+1}^{i-1} F_{p}$, 从而存在 $\left\{F_{r_{1}+1}, \ldots, F_{i-1}\right\}$ 中之一, 记为 $F_{i-1}$, 它包含 $G$ 中至少 3 条边 $b_{1} b_{2}, b_{3} b_{4}$ 和 $b_{5} b_{6}$, 且这 些边在 $G$ 中只与 $B$ 的顶点相关联, 则 $\left\{b_{1}, b_{2}, y_{i-1}, z_{i-1}\right\} \subset B$. 因为 $|B|=\frac{n-1}{2}$, 所以 $\left|F_{i-1} \cap E(G[A])\right| \geqslant 2$, 从而假设 $a_{1} a_{2}, a_{3} a_{4} \in F_{i-1} \cap E(G[A])$. 设 $G_{i-1}=G-\left(E_{1} \cup F_{1} \cup \cdots \cup F_{i-2}\right)$, $G_{i-1}^{\prime}=G_{i-1}-\left\{b_{1} b_{2}\right\}$ 和 $H_{i-1}^{\prime}=G_{i-1}^{\prime}-\left\{y_{i-1}, z_{i-1}\right\}$. 类似地, 有 $\Delta\left(G_{i-1}\right)=\delta\left(G_{i-1}\right)+1$, 因 此 $\Delta\left(G_{i-1}\right)-2 \leqslant \delta\left(G_{i-1}^{\prime}\right) \leqslant \Delta\left(G_{i-1}^{\prime}\right) \leqslant \Delta\left(G_{i-1}\right)$, 且 $G_{i-1}^{\prime}$ 有至多两个度为 $\Delta\left(G_{i-1}\right)-2$ 的 顶点 $\left(b_{1}\right.$ 和 $\left.b_{2}\right)$.

因为 $B$ 是 $H_{i}$ 的一个独立集, 以及 $\left\{b_{1}, b_{2}, y_{i-1}, z_{i-1}\right\} \subset B$, 所以, 在 $H_{i}$ 中, $y_{i-1}$ 和 $z_{i-1}$ 都 不与 $b_{1}$ 和 $b_{2}$ 相邻. 因为在 $G$ 中, $y_{i-1}$ 和 $z_{i-1}$ 不与 $F_{i-1}$ 的边相关联, 并且 $b_{1} b_{2} \subset F_{i-1}$, 所以, 在 $G_{i-1}$ 中, $y_{i-1}$ 和 $z_{i-1}$ 都不与 $b_{1}$ 和 $b_{2}$ 相邻. 因此, 对于 $v \in\left\{b_{1}, b_{2}\right\}, d_{H_{i-1}^{\prime}}(v) \geqslant \Delta\left(G_{i-1}\right)-2$; 对于 $v \in V\left(H_{i-1}^{\prime}\right) \backslash\left\{b_{1}, b_{2}\right\}, d_{H_{i-1}^{\prime}}(v) \geqslant \Delta\left(G_{i-1}\right)-3$. 于是

$$
\Delta\left(G_{i-1}\right)-3 \leqslant \delta\left(H_{i-1}^{\prime}\right) \leqslant \Delta\left(H_{i-1}^{\prime}\right) \leqslant \Delta\left(G_{i-1}\right) .
$$


设 $\Delta_{1}=\Delta\left(G_{i-1}\right)$, 则 $G_{i-1}^{\prime}$ 是 $\left(\Delta_{1}-2, \Delta_{1}\right)$-图且 $H_{i-1}^{\prime}$ 是 $\left(\Delta_{1}-3, \Delta_{1}\right)$-图. 由 (5) 式, $\Delta_{1}-3 \geqslant$ $\Delta-3 \geqslant \frac{n}{3}+8$. 因为 $\delta\left(G_{i}\right) \geqslant \frac{n}{3}+10$ 和 $G_{i}-\left\{x_{i}, y_{i}\right\}$ 有一个独立集 $B$ 满足 $|B|=\frac{n-1}{2}$, 所以, 由推论 $1, G_{i}-\left\{y_{i-1}, z_{i-1}\right\}$ 是 2 - 连通的. 因为 $G_{i}-\left\{y_{i-1}, z_{i-1}\right\}=H_{i-1}^{\prime}-F_{i-1}$, 所以, $H_{i-1}^{\prime}$ 也 是 2 - 连通的. 因为 $G_{i}$ 有至多 $\frac{n}{3}$ 个度为 $\Delta\left(G_{i}\right)$ 的顶点, 所以, $G_{i-1}^{\prime}$ 有至多 $\frac{n}{3}$ 个度为 $\Delta_{1}$ 的顶 点. 假设 $H_{i-1}^{\prime}$ 不是 Hamilton 图, 则 $G_{i-1}^{\prime}$ 和 $H_{i-1}^{\prime}$ 都满足引理 4 的假设, 从而存在 $V\left(H_{i-1}^{\prime}\right)$ 的一个子集 $A_{i-1}$, 使得 $\left|A_{i-1}\right|=m-1$ 和 $\omega\left(H_{i-1}^{\prime}-A_{i-1}\right)=m$. 设 $B_{i-1}=V\left(H_{i-1}^{\prime}-A_{i-1}\right)$, 则 $B_{i-1}$ 是 $H_{i-1}^{\prime}$ 的独立集且 $\left|B_{i-1}\right|=m$.

若 $B \cap B_{i-1}=\emptyset$. 因为 $A \cap B=\emptyset,|V(G)|=2 m+1,|B|=\left|B_{i-1}\right|=m$ 和 $|A|=m-1$, 所 以, 存在至多一个顶点 $u \in A$, 使得 $u \notin B_{i-1}$. 因为 $a_{1} a_{2}, a_{3} a_{4} \in F_{i-1} \cap E(G[A]) \subset E\left(H_{i-1}^{\prime}\right)$, 所以, 存在 $\left\{a_{1} a_{2}, a_{3} a_{4}\right\}$ 中的一条边, 记为 $a_{1} a_{2}$, 使得 $a_{1} a_{2} \in E\left(H_{i-1}^{\prime}-A_{i-1}\right)$. 所以 $B_{i-1}$ 不 是 $H_{i-1}^{\prime}$ 的一个独立集, 矛盾. 因而, $B \cap B_{i-1} \neq \emptyset$. 因为 $y_{i-1}, z_{i-1} \in B$, 如同 (8) 式, 所以有

$$
\left|A \cap A_{i-1}\right| \geqslant \delta\left(H_{i}\right) \geqslant \frac{n}{3}+8 .
$$

情形 1.3.1 若 $\left|A \backslash A_{i-1}\right| \leqslant 1$. 如果 $\left|A \backslash A_{i-1}\right|=0$, 因为 $|A|=m-1=\left|A_{i-1}\right|$, 所以 $A=$ $A_{i-1}$. 因为 $y_{i-1}, z_{i-1} \in B$ (注意 $B$ 和 $B_{i-1}$ 的定义), 所以 $B_{i-1}=\left(B \backslash\left\{y_{i-1}, z_{i-1}\right\}\right) \cup\left\{x_{i}, y_{i}\right\}$. 由断言 1 , 在 $G_{i}$ 中, $x_{i}$ 至少与 $B \backslash\left\{y_{i-1}, z_{i-1}\right\}$ 的 3 个顶点相邻, 则在 $H_{i-1}^{\prime}$ 中, $x_{i}$ 至少与 $B \backslash\left\{y_{i-1}, z_{i-1}\right\}$ 的 3 个顶点相邻. 这与 $B_{i-1}$ 是 $H_{i-1}^{\prime}$ 的一个独立集相矛盾. 如果 $\left|A \backslash A_{i-1}\right|=1$, 则类似地, $x_{i} \in B_{i-1}$ 或者 $y_{i} \in B_{i-1}$, 可以得到矛盾.

情形 1.3.2 若 $2 \leqslant\left|A \backslash A_{i-1}\right| \leqslant 3$. 由引理 $6,\left|B \backslash A_{i-1}\right| \geqslant m-3$, 则 $\mid B \backslash\left(A_{i-1} \cup\right.$ $\left.\left\{y_{i-1}, z_{i-1}\right\}\right) \mid \geqslant m-5$. 因为 $y_{i-1}, z_{i-1} \notin A$ 和 $\left|A \backslash A_{i-1}\right| \geqslant 2$ 以及 (9) 式, 所以存在 $u, v \in$ $A \backslash A_{i-1} \subset B_{i-1}$, 使得

$$
\begin{aligned}
e_{H_{i}}(\{u, v\}, B) & \geqslant 2 \delta\left(H_{i}\right)-\left(\delta\left(H_{i}\right)-3\right)-1 \\
& \left.=\delta\left(H_{i}\right)+2 \geqslant \frac{n}{3}+10 \geqslant 28 \text { (因为 } n \geqslant 55\right),
\end{aligned}
$$

则 $e_{H_{i}}\left(\{u, v\}, B \backslash\left(A_{i-1} \cup\left\{y_{i-1}, z_{i-1}\right\}\right)\right) \geqslant 28-10=18$, 并且

$$
e_{H_{i}-\left\{y_{i-1}, z_{i-1}\right\}}\left(\{u, v\}, B \backslash\left(A_{i-1} \cup\left\{y_{i-1}, z_{i-1}\right\}\right)\right) \geqslant 18 .
$$

因为 $H_{i}-\left\{y_{i-1}, z_{i-1}\right\}$ 是 $H_{i-1}^{\prime}$ 的一个子图, 所以

$$
e_{H_{i-1}^{\prime}}\left(\{u, v\}, B \backslash\left(A_{i-1} \cup\left\{y_{i-1}, z_{i-1}\right\}\right)\right) \geqslant 18 .
$$

因为 $u, v \in B_{i-1}$ 和 $B \backslash\left(A_{i-1} \cup\left\{y_{i-1}, z_{i-1}\right\}\right) \subset B_{i-1}$, 所以 $B_{i-1}$ 不是 $H_{i-1}^{\prime}$ 的一个独立集, 矛 盾.

情形 1.3.3 若 $\left|A \backslash A_{i-1}\right| \geqslant 4$. 如同情形 1.1.3, 存在一个顶点 $v \in A \backslash A_{i-1}$, 使得

$$
\begin{aligned}
e_{H_{i-1}^{\prime}}\left(\{v\}, B \backslash\left(A_{i-1} \cup\left\{y_{i-1}, z_{i-1}\right\}\right)\right) & \geqslant e_{H_{i-1}^{\prime}}\left(\{v\}, B \backslash\left(\left(B \cap A_{i-1}\right) \cup\left\{y_{i-1}, z_{i-1}\right\}\right)\right) \\
& \geqslant\left(\frac{n}{6}+4\right)-\left(\frac{n}{6}-\frac{19}{2}\right)-2 \geqslant 11 .
\end{aligned}
$$

因为 $v \in A \backslash A_{i-1} \subset B_{i-1}$ 和 $B \backslash\left(A_{i-1} \cup\left\{y_{i-1}, z_{i-1}\right\}\right) \subset B_{i-1}$, 所以 $B_{i-1}$ 不是 $H_{i-1}^{\prime}$ 的一个 独立集, 矛盾.

因此, $H_{i-1}^{\prime}$ 是 Hamilton 图. 设 $C_{i-1}^{\prime}$ 是 $H_{i-1}^{\prime}$ 中的一个 Hamilton 圈并且设 $F_{i-1}^{\prime}$ 是 $H_{i-1}^{\prime}$ 中的一个最大匹配, 使得 $F_{i-1}^{\prime} \subset E\left(C_{i-1}^{\prime}\right)$ 且 $x_{i-1}$ 不与 $F_{i-1}^{\prime}$ 中的边相关联. 
设 $G_{i}^{\prime}=G-\left(E_{1} \cup F_{1} \cup \cdots \cup F_{i-2} \cup F_{i-1}^{\prime}\right)$ 和 $H_{i}^{\prime}=G_{i}^{\prime}-\left\{x_{i}, y_{i}\right\}$, 则 $\Delta=\Delta\left(G_{i}\right)=\Delta\left(G_{i}^{\prime}\right)$. 类似地, 有 $(7)$ 式, $G_{i}^{\prime}$ 是 $(\Delta-1, \Delta)$-图, $H_{i}^{\prime}$ 是 $(\Delta-3, \Delta)$-图, 以及 $G_{i}^{\prime}$ 有至多 $\frac{n}{3}$ 个度为 $\Delta$ 的 顶点. 由 (4) 式, $\delta\left(H_{i}-F_{i-1}^{\prime}\right) \geqslant \frac{n}{3}+7$. 因为 $B$ 是 $H_{i}$ 的一个独立集, 所以 $B$ 也是图 $H_{i}-F_{i-1}^{\prime}$ 的一个独立集. 因为 $n \geqslant 55$, 所以 $|B|=\frac{n-1}{2} \geqslant \frac{n}{3}+1$. 由引理 5 , 图 $H_{i}-F_{i-1}^{\prime}$ 是 2 - 连通的. 因为 $H_{i}^{\prime}=\left(H_{i}-F_{i-1}^{\prime}\right)+\left(F_{i-1} \backslash F_{i-1}^{\prime}\right)$, 所以 $H_{i}^{\prime}$ 也是 2 - 连通的. 假设 $H_{i}^{\prime}$ 不是 Hamilton 图. 则 $G_{i}^{\prime}$ 和 $H_{i}^{\prime}$ 都满足引理 4 的假设, 从而存在 $V\left(H_{i}^{\prime}\right)$ 的一个子集 $A^{\prime}$, 使得 $\left|A^{\prime}\right|=m-1$ 和 $\omega\left(H_{i}^{\prime}-A^{\prime}\right)=m$. 设 $B^{\prime}=V\left(H_{i}^{\prime}-A^{\prime}\right)$, 则 $B^{\prime}$ 是 $H_{i}^{\prime}$ 的独立集且 $\left|B^{\prime}\right|=m$.

若 $B \cap B^{\prime}=\emptyset$, 则 $B^{\prime} \subset A$. 因为 $\left|B^{\prime}\right|=m$, 这与 $|A|=m-1$ 相矛盾. 因此 $B \cap B^{\prime} \neq \emptyset$, 从 而存在一个顶点 $u \in B \cap B^{\prime}$, 使得在 $H_{i}$ 中, $u$ 至少与 $A$ 的 $\delta\left(H_{i}\right)$ 个顶点相邻, 则在 $H_{i}-F_{i-1}^{\prime}$ 中, $u$ 至少与 $\mathrm{A}$ 的 $\delta\left(H_{i}\right)-1$ 个顶点相邻. 因为 $H_{i}-F_{i-1}^{\prime}$ 是 $H_{i}^{\prime}$ 的子图, $B^{\prime}$ 是 $H_{i}^{\prime}$ 的独立集, 所以, 在 $H_{i}^{\prime}$ 中, $u$ 至少与 $A \cap A^{\prime}$ 的 $\delta\left(H_{i}\right)-1$ 个顶点相邻, 则

$$
\left|A \cap A^{\prime}\right| \geqslant \delta\left(H_{i}\right)-1 \geqslant \frac{n}{3}+7 .
$$

情形 1.3.4 若 $\left|A \backslash A^{\prime}\right|=0$, 则 $A=A^{\prime}$ 且 $b_{1}, b_{2} \in B^{\prime}$, 从而 $b_{1} b_{2} \in E\left(H_{i}^{\prime}-A^{\prime}\right)$. 然而, 这 与 $B^{\prime}$ 是 $H_{i}^{\prime}$ 的独立集相矛盾.

情形 1.3.5 若 $1 \leqslant\left|A \backslash A^{\prime}\right| \leqslant 3$, 这与情形 1.1 .2 相类似, 则可以得到矛盾.

情形 1.3.6 若 $\left|A \backslash A^{\prime}\right| \geqslant 4$, 如同情形 1.1.3, 则可以得到矛盾.

因此, $H_{i}^{\prime}$ 是 Hamilton 图. 设 $C_{i}^{\prime}$ 是 $H_{i}^{\prime}$ 中的一个 Hamilton 圈并且设 $F_{i}$ 是 $H_{i}^{\prime}$ 中的最大 匹配, 使得 $F_{i} \subset E\left(C_{i}^{\prime}\right)$ 且 $z_{i}$ 不与 $F_{i}$ 中的边相关联. 我们用 $F_{i-1}^{\prime}$ 替代 $F_{i-1}$.

结合情形 1.1-1.3, 对于情形 1 时, 引理成立.

情形 $2 \quad H_{i}$ 是 1-连通的, 但不是 2-连通的.

设 $w$ 是 $H_{i}$ 的一个割点. 由 (4) 式,

$$
\delta\left(H_{i}-\{w\}\right) \geqslant \frac{n}{3}+7 .
$$

因此, $H_{i}-\{w\}$ 恰好包含两个分支, 记为 $H_{i}^{(1)}$ 和 $H_{i}^{(2)}$. 由 (15) 式,

且

$$
\min \left\{\delta\left(H_{i}^{(1)}\right), \delta\left(H_{i}^{(2)}\right)\right\} \geqslant \frac{n}{3}+7
$$

$$
\min \left\{\left|V\left(H_{i}^{(1)}\right)\right|,\left|V\left(H_{i}^{(2)}\right)\right|\right\} \geqslant \frac{n}{3}+8
$$

因此

$$
\max \left\{\left|V\left(H_{i}^{(1)}\right)\right|,\left|V\left(H_{i}^{(2)}\right)\right|\right\} \leqslant n-\left(\frac{n}{3}+8\right) \leqslant \frac{2 n}{3}-8 .
$$

情形 2.1 若 $w \neq z_{i}$. 不失一般性, 假设 $z_{i} \in V\left(H_{i}^{(2)}\right)$. 由 (16) 和 (18) 式, 以及由 Dirac 定理, 图 $H_{i}^{(1)}$ 和 $H_{i}^{(2)}-\left\{z_{i}\right\}$ 是 Hamilton 图. 设 $C_{i}^{(1)}=u_{0} u_{1} \cdots u_{p} u_{0}$ 是 $H_{k}^{(1)}$ 中的一 个 Hamilton 圈, $C_{i}^{(2)}=v_{0} v_{1} \cdots v_{q} v_{0}$ 是 $H_{i}^{(2)}-\left\{z_{i}\right\}$ 中的一个 Hamilton 圈. 不失一般性, 假设 在 $H_{i}$ 中, $w$ 与两个顶点 $u_{0}$ 和 $v_{0}$ 相邻, 则 $P=u_{1} \cdots u_{p} u_{0} w v_{0} v_{1} \cdots v_{q}$ 是 $H_{i}-\left\{z_{i}\right\}$ 中的一个 Hamilton 路. 设 $F_{i}$ 是 $H_{i}-\left\{z_{i}\right\}$ 中的最大匹配, 使得 $F_{i} \subset E(P)$, 则 $F_{i}$ 不饱和 $x_{i}, y_{i}$ 和 $z_{i}$, 而饱和 $G$ 的其他的顶点. 
情形 2.2 若 $w=z_{i}$.

由 (4) 式, 容易得到 $H_{i}$ 有至多两个割点. 因为 $i \leqslant k$ 和 (1) 式, 所以 $3 i \leqslant 3 k \leqslant n-\frac{33}{2}$, 从而 $\left|\left\{z_{i+1}, \ldots, z_{l}\right\}\right| \geqslant 3$, 因此 $\left\{z_{i+1}, \ldots, z_{l}\right\}$ 中存在一个顶点, 它不是 $H_{i}$ 的割点. 不失一般 性, 假设 $z_{i+1}$ 不是 $H_{i}$ 中的一个割点. 我们用 $z_{i+1}$ 替换 $z_{i}$, 如同情形 2.1 , 引理成立.

情形 $3 \quad H_{i}$ 是非连通的.

由 (4) 式, $H_{i}$ 恰好包含两个分支, 记为 $H_{i}^{(1)}$ 和 $H_{i}^{(2)}$. 我们有

以及

$$
\begin{aligned}
& \min \left\{\delta\left(H_{i}^{(1)}\right), \delta\left(H_{i}^{(2)}\right)\right\} \geqslant \frac{n}{3}+8, \\
& \min \left\{\left|V\left(H_{i}^{(1)}\right)\right|,\left|V\left(H_{i}^{(2)}\right)\right|\right\} \geqslant \frac{n}{3}+9,
\end{aligned}
$$

$$
\max \left\{\left|V\left(H_{i}^{(1)}\right)\right|,\left|V\left(H_{i}^{(2)}\right)\right|\right\} \leqslant n-\left(\frac{n}{3}+9\right) \leqslant \frac{2 n}{3}-9 .
$$

我们可以假设 $\left.\left|V\left(H_{i}^{(1)}\right)\right| \leqslant \mid V\left(H_{i}^{(2)}\right)\right) \mid$, 则 $\left|V\left(H_{i}^{(1)}\right)\right| \leqslant \frac{n-1}{2}$, 从而

$$
\begin{aligned}
e_{G}\left(V\left(H_{i}^{(1)}\right), V\left(H_{i}^{(2)}\right)\right) & \geqslant\left|V\left(H_{i}^{(1)}\right)\right|\left(d(G)-2-\Delta\left(G\left[V\left(H_{i}^{(1)}\right)\right]\right)\right) \\
& \geqslant\left|V\left(H_{i}^{(1)}\right)\right|\left(d(G)-2-\left(\left|V\left(H_{i}^{(1)}\right)\right|-1\right)\right) \\
& \geqslant\left(\frac{n}{3}+9\right)\left(\frac{2 n}{3}+\frac{7}{2}-2-\left(\frac{n-1}{2}-1\right)\right) \text { (由 }(2) \text { 和 }(20) \text { 式) } \\
& \geqslant\left(\frac{n}{3}+9\right)\left(\frac{n}{6}+3\right) .
\end{aligned}
$$

因为 $i \leqslant k$ 和 $\left|E_{1}\right|=r \leqslant \frac{n}{6}+2$, 所以

$$
\begin{aligned}
& \frac{\mid E\left(G\left[V\left(H_{i}^{(1)}, V\left(H_{i}^{(2)}\right)\right]\right) \backslash E_{1} \mid\right.}{i-1} \geqslant \frac{\left(\frac{n}{3}+9\right)\left(\frac{n}{6}+3\right)-\left(\frac{n}{6}+2\right)}{k} \text { (由 (1) 式) } \\
&\left.\geqslant \frac{\frac{n^{2}}{18}}{\frac{n}{3}-\frac{11}{2}} \geqslant \frac{n}{6}>9 \text { (因为 } n \geqslant 55\right) .
\end{aligned}
$$

因为 $H_{i}$ 恰好包含两个分支 $H_{i}^{(1)}$ 和 $H_{i}^{(2)}$, 所以 $E\left(G\left[V\left(H_{i}^{(1)}\right), V\left(H_{i}^{(2)}\right)\right]\right) \backslash E_{1} \subset \bigcup_{j=1}^{i-1} F_{j}$, 于 是, 至少存在 $\left\{F_{1}, \ldots, F_{i-1}\right\}$ 中一个匹配, 记为 $F_{i-1}, F_{i-1}$ 至少包含 $\left(G-\left\{x_{i}, y_{i}\right\}\right)-\left(E_{1} \cup\right.$ $\left.E\left(G\left[V\left(H_{i}^{(1)}\right)\right]\right) \cup E\left(G\left[V\left(H_{i}^{(2)}\right)\right]\right)\right)$ 中的 9 条边. 因为 $F_{i-1}$ 不饱和 $x_{i-1}, y_{i-1}$ 和 $z_{i-1}$, 所以可 以假设 $a_{j} b_{j} \in F_{i-1}$, 使得 $a_{j} \in V\left(H_{i}^{(1)}\right)$ 和 $b_{j} \in V\left(H_{i}^{(2)}\right)$, 以及 $a_{j}, b_{j} \notin\left\{x_{i-1}, y_{i-1}, z_{i-1}, x_{i}\right.$, $\left.y_{i}\right\}$ 对于 $j=1,2$. 因为 $\delta\left(G_{i}\right) \geqslant \frac{n}{3}+10>10$, 所以 $G_{i}$ 包含一个匹配 $\left\{x_{i} u, y_{i} v\right\}$, 使得 $u, v \notin\left\{x_{i-1}, y_{i-1}, z_{i-1}, a_{1}, b_{1}\right\}$. 设 $V_{5}=\left\{x_{i-1}, y_{i-1}, z_{i-1}, x_{i}, y_{i}, u, v\right\}$. 由 (19) 和 (21) 式以 及 Dirac 定理, 图 $H_{i}^{(1)}-V_{5}$ 和 $H_{i}^{(2)}-V_{5}$ 是 Hamilton 图. 设 $C_{i-1}^{(1)}=a_{1} u_{1} \cdots u_{p} a_{1}$ 是 $H_{i}^{(1)}-V_{5}$ 中的一个 Hamilton 圈, $C_{i-1}^{(2)}=b_{1} v_{1} \cdots v_{q} b_{1}$ 是 $H_{i}^{(2)}-V_{5}$ 中的一个 Hamilton 圈. 则 $P=u_{1} \cdots u_{p} a_{1} b_{1} v_{1} \cdots v_{q}$ 是图 $\left(H_{i}+\left\{a_{1} b_{1}\right\}\right)-V_{5}$ 中的 Hamilton 路. 设 $M_{i-1}$ 是图 $\left(H_{i}+\left\{a_{1} b_{1}\right\}\right)-V_{5}$ 中的最大匹配, 使得 $M_{i-1} \subset E(P)$. 设 $F_{i-1}^{\prime}=M_{i-1} \cup\left\{x_{i} u, y_{i} v\right\}$, 则 $G$ 包含一个匹配 $F_{i-1}^{\prime}$, 使得 $F_{i-1}^{\prime}$ 不饱和 $x_{i-1}, y_{i-1}$ 和 $z_{i-1}$, 而饱和 $G$ 的其他顶点. 类似地, 图 $\left(H_{i}^{(1)}-F_{i-1}^{\prime}\right)-\left\{z_{i}\right\}$ 和 $\left(H_{i}^{(2)}-F_{i-1}^{\prime}\right)-\left\{z_{i}\right\}$ 是 Hamilton 图. 设 $C_{i}^{(1)}=a_{2} u_{1}^{\prime} \cdots u_{s}^{\prime} a_{2}$ 是图 $\left(H_{i}^{(1)}-F_{i-1}^{\prime}\right)-\left\{z_{i}\right\}$ 中的 Hamilton 圈, $C_{i}^{(2)}=b_{2} v_{1}^{\prime} \cdots v_{t}^{\prime} b_{2}$ 是图 $\left(H_{i}^{(2)}-F_{i-1}^{\prime}\right)-\left\{z_{i}\right\}$ 中的 
Hamilton 圈. 则 $P^{\prime}=u_{1}^{\prime} \cdots u_{s}^{\prime} a_{2} b_{2} v_{1}^{\prime} \cdots v_{t}^{\prime}$ 是图 $\left(\left(H_{i}+\left\{a_{2} b_{2}\right\}\right)-F_{i-1}^{\prime}\right)-\left\{z_{i}\right\}$ 中的 Hamilton 路. 设 $F_{i}$ 是图 $\left(\left(H_{i}+\left\{a_{2} b_{2}\right\}\right)-F_{i-1}^{\prime}\right)-\left\{z_{i}\right\}$ 中的最大匹配, 使得 $F_{i} \subset E\left(P^{\prime}\right)$, 则 $F_{i}$ 不饱和 $x_{i}, y_{i}$ 和 $z_{i}$, 而饱和所有其他的顶点. 我们用 $F_{i-1}^{\prime}$ 替换 $F_{i-1}$.

结合情形 $1-3$, 引理成立.

引理 10 存在 $G$ 的边不相交的匹配 $F_{1}, \ldots, F_{k}$, 使得对于 $1 \leqslant i \leqslant k, F_{i}$ 不饱和 $x_{i}, y_{i}$ 和 $z_{i}$, 而饱和 $\mathrm{G}$ 的所有其他的顶点.

证明 对于 $k \leqslant\left\lfloor\frac{2 n}{9}\right\rfloor+1$, 由引理 9 , 结论成立. 假设对于 $i-1 \geqslant\left\lfloor\frac{2 n}{9}\right\rfloor+1$ 结论成立, 即 存在 $G$ 的边不相交的匹配 $F_{1}, \ldots, F_{i-1}$, 使得对于 $1 \leqslant j \leqslant i-1 \leqslant k-1, F_{j}$ 不饱和 $x_{j}, y_{j}$ 和 $z_{j}$, 而饱和 $G$ 的所有其他的顶点. 设 $G_{i}=G-\left(F_{1} \cup \cdots \cup F_{i-1}\right)$ 和 $H_{i}=G_{i}-\left\{x_{i}, y_{i}\right\}$. 设 $V_{2}=\left\{x_{1}, y_{1}, z_{1}, \ldots, x_{i-1}, y_{i-1}, z_{i-1}\right\}$. 因为 $G$ 是正则图, 所以, 对于 $u \in V_{2}$, 有 $d_{G_{i}}(u)=\Delta\left(G_{i}\right) ;$ 对于 $\left.u \in V\left(G_{i}\right)\right) \backslash V_{2}$, 有 $d_{G_{i}}(u)=\Delta\left(G_{i}\right)-1=\delta\left(G_{i}\right)$, 因此 $\Delta\left(G_{i}\right)=\delta\left(G_{i}\right)+1$. 类似地, 我 们有 (3)-(5) 式. 设 $\Delta=\Delta\left(G_{i}\right)$, 则 $G_{i}$ 是 $(\Delta-1, \Delta)$ - 图, 并且 $H_{i}$ 是 $(\Delta-3, \Delta)$-图, 以及 $\Delta-3 \geqslant \frac{n}{3}+8$.

情形 $1 \quad H_{i}$ 是 2-连通的.

若 $H_{i}$ 是 Hamilton 图. 如同引理 8 , 结论成立. 现在假设 $H_{i}$ 是非 Hamilton 图. 因为

$$
\left|V_{2}\right|=3(i-1) \geqslant 3\left(\left\lfloor\frac{2 n}{9}\right\rfloor+1\right) \geqslant 3\left(\frac{2 n}{9}\right)=\frac{2 n}{3},
$$

所以 $G_{i}$ 有至少 $\frac{2 n}{3}$ 个度为 $\Delta$ 的顶点. 则 $G_{i}$ 和 $H_{i}$ 满足引理 4 的假设, 那么存在 $V\left(H_{i}\right)$ 的一个子集 $A$, 使得 $|A|=m-1$ 并且 $\omega\left(H_{i}-A\right)=m$. 设 $B=V\left(H_{i}-A\right)$, 则 $B$ 是 $H_{i}$ 的一 个独立集并且 $|B|=m$.

断言 4 在 $G_{i}$ 中, $x_{i}$ 和 $y_{i}$ 都与 $B$ 的至少 5 个顶点相邻.

证明 假设结论不成立. 不失一般性, 假设在 $G_{i}$ 中, $y_{i}$ 至多与 $B$ 中 4 个顶点相邻. 则在 $G_{i}$ 中, $y_{i}$ 至少与 $A \cup\left\{x_{i}\right\}$ 中的 $\delta\left(G_{i}\right)-4$ 个顶点相邻. 因为 $G_{i}$ 至少有 $\frac{2 n}{3}$ 个度为 $\Delta$ 的顶点, 所以 $G_{i}$ 至多有 $\frac{n}{3}$ 个度为 $\delta\left(G_{i}\right)$ 的顶点. 因为 $B$ 是 $H_{i}$ 的一个独立集, 以及 $\left|B \cup\left\{y_{i}\right\}\right|=m+1$, 所以在 $G_{i}$ 中, 连接 $B \cup\left\{y_{i}\right\}$ 与 $A \cup\left\{x_{i}\right\}$ 的顶点的边数至少是 $\left(\delta\left(G_{i}\right)+1\right)(m+1)-8-\frac{n}{3}$, 也就是

$$
e_{G_{i}}\left(B \cup\left\{y_{i}\right\}, A \cup\left\{x_{i}\right\}\right) \geqslant\left(\delta\left(G_{i}\right)+1\right)(m+1)-8-\frac{n}{3} .
$$

另一方面, 因为 $\Delta\left(G_{i}\right)=\delta\left(G_{i}\right)+1$ 和 $\left|A \cup\left\{x_{i}\right\}\right|=m$, 所以在 $G_{i}$ 中, 连接 $A \cup\left\{x_{i}\right\}$ 与 $B \cup\left\{y_{i}\right\}$ 的顶点的边数至多是 $\left(\delta\left(G_{i}\right)+1\right) m$, 也就是

$$
e_{G_{i}}\left(A \cup\left\{x_{i}\right\}, B \cup\left\{y_{i}\right\}\right) \leqslant\left(\delta\left(G_{i}\right)+1\right) m .
$$

因此 $\left(\delta\left(G_{i}\right)+1\right) m \geqslant\left(\delta\left(G_{i}\right)+1\right)(m+1)-8-\frac{n}{3}$, 那么 $\frac{n}{3}+7 \geqslant \delta\left(G_{i}\right)$. 这与 (5) 式矛盾, 则断 言成立.

类似地, 引理 9 的断言 2 和 3 也成立.

情形 1.1 若 $B \cap\left\{z_{i}, \ldots, z_{k}, \ldots, z_{l}\right\} \neq \emptyset$.

除引理 9 的情形 1.1.3, 其他的证明如同引理 9 的情形 1.1 .

情形 1.1.1 若 $\left|A \backslash A^{\prime}\right| \geqslant 4$. 设 $p=\left|A \backslash A^{\prime}\right|$. 因为 $B$ 是 $H_{i}$ 的一个独立集, $G_{i}$ 至多有 $\frac{n}{3}$ 个度为 $\delta\left(G_{i}\right)$ 的顶点, 以及 $d_{G_{i}}\left(x_{i}\right)=\delta\left(G_{i}\right)=d_{G_{i}}\left(y_{i}\right)$, 所以在 $H_{i}$ 中, 连接 $B$ 与 $A$ 的顶点的 
边数至少是 $\delta\left(G_{i}\right)|B|+\left(|B|-\frac{n}{3}\right)-2 \delta\left(G_{i}\right)$. 因为 $\Delta\left(H_{i}\right) \leqslant \Delta\left(G_{i}\right) \leqslant \delta\left(G_{i}\right)+1$ 和 $|A|=m-1$, 所以

$$
\begin{aligned}
\left|E\left(H_{i}[A]\right)\right| & \leqslant \frac{\left(\delta\left(G_{i}\right)+1\right)(m-1)-\left(\delta\left(G_{i}\right)|B|+\left(|B|-\frac{n}{3}\right)-2 \delta\left(G_{i}\right)\right)}{2} \\
& =\frac{1}{2} \delta\left(G_{i}\right)+\frac{n}{6}-\frac{1}{2} \quad(\text { 因为 }|B|=m, \text { 如同 }(9) \text { 式) } \\
& \leqslant \delta\left(H_{i}\right)-3 .
\end{aligned}
$$

如同引理 9 的情形 1.1.3 的证明, 引理成立.

情形 1.2 若 $B \cap\left\{z_{i}, \ldots, z_{k}, \ldots, z_{l}\right\}=\emptyset$, 但 $B \cap\left\{x_{i+1}, y_{i+1}, \ldots, x_{k}, y_{k}\right\} \neq \emptyset$.

如同引理 9 的情形 1.2 的证明, 引理成立.

情形 1.3 若 $B \cap\left\{x_{i+1}, \ldots, x_{k}, y_{i+1}, \ldots, y_{k}, z_{i}, \ldots, z_{k}, \ldots, z_{l}\right\}=\emptyset$.

设 $V_{3}=\left\{x_{i+1}, \ldots, x_{k}, y_{i+1}, \ldots, y_{k}, z_{i}, \ldots, z_{k}, \ldots, z_{l}\right\}$, 则 $V_{3} \subset A$, 从而 $B \subset V_{2}$. 因为 $i \leqslant k$, 由 (1) 式, 得

因为

$$
\left|V_{3}\right|=|V(G)|-3 i+1 \geqslant n-3 k+1 \geqslant n-3\left(\frac{n}{3}-\frac{11}{2}\right)+1=\frac{35}{2} .
$$

$$
|A|=\frac{n-1}{2}-1
$$

所以

$$
\left|A \backslash V_{3}\right|=|A|-\left|V_{3}\right| \leqslant\left(\frac{n-1}{2}-1\right)-\frac{35}{2}=\frac{n}{2}-19 .
$$

设 $r_{2}=\left\lfloor\frac{1}{3}\left(\frac{n}{2}-19\right)\right\rfloor$, 则 $\left\{F_{1}, \ldots, F_{i-1}\right\}$ 包含 $r_{2}$ 个匹配, 记为 $F_{1}, \ldots, F_{r_{2}}$, 使得 $\left\{x_{1}, y_{1}, z_{1}, \ldots\right.$, $\left.x_{r_{2}}, y_{r_{2}}, z_{r_{2}}\right\} \subset A$. 因为 $r_{2}<i-1$, 所以 $\left\{x_{j}, y_{j}, z_{j}\right\} \cap B \neq \emptyset$, 对于 $j=r_{2}+1, \ldots, i-1$, 有

$$
\begin{aligned}
\left|E(G[B]) \cap \bigcup_{j=1}^{r_{2}} F_{j}\right| & \leqslant \frac{1}{2} r_{2}|B| \leqslant \frac{1}{2}\left|\frac{1}{3}\left(\frac{n}{2}-19\right)\right|\left(\frac{n-1}{2}\right) \\
& \leqslant \frac{1}{2}\left(\frac{n}{6}-\frac{19}{3}\right)\left(\frac{n-1}{2}\right),
\end{aligned}
$$

因此, 由 (13) 式, 可得

$$
\begin{aligned}
\left|E(G[B]) \backslash \bigcup_{j=1}^{r_{2}} F_{j}\right| & \geqslant|E(G[B])|-\left|E(G[B]) \cap \bigcup_{j=1}^{r_{2}} F_{j}\right| \\
& \geqslant \frac{1}{2}\left(\frac{n}{6}+4\right) \frac{n-1}{2}-\frac{1}{2}\left(\frac{n}{6}-\frac{19}{3}\right)\left(\frac{n-1}{2}\right) \\
& =\frac{1}{2}\left(\frac{n-1}{2}\right)\left(4+\frac{19}{3}\right) \geqslant \frac{7}{3}(n-1) .
\end{aligned}
$$

因为 $r_{2}<i-1 \leqslant k-1$, 所以

$$
\begin{aligned}
\frac{\left|E(G[B]) \backslash \bigcup_{j=1}^{r_{2}} F_{j}\right|}{i-1-r_{2}} & \geqslant \frac{7(n-1)}{k-1-r_{2}} \geqslant \frac{\frac{7(n-1)}{3}}{\left(\frac{n}{3}-\frac{11}{2}\right)-1-\left\lceil\frac{1}{3}\left(\frac{n}{2}-19\right)\right\rceil} \quad \text { (由 (1) 式) } \\
& \geqslant \frac{\frac{7(n-1)}{3}}{\left(\frac{n}{3}-\frac{11}{2}\right)-1-\left(\frac{n}{6}-\frac{19}{3}\right)} \geqslant \frac{\frac{7(n-1)}{3}}{\frac{n}{6}-\frac{1}{6}}=14 .
\end{aligned}
$$


因为 $B$ 是 $H_{i}$ 的一个独立集, 所以 $E(G[B]) \backslash \bigcup_{j=1}^{r_{2}} F_{j} \subset \bigcup_{p=r_{2}+1}^{i-1} F_{p}$, 从而存在 $\left\{F_{r_{2}+1}, \ldots, F_{i-1}\right\}$ 中的至少一个, 记为 $F_{i-1}$, 它包含 $G$ 的至少 14 条边 $b_{1} b_{2}, \ldots, b_{27} b_{28}$, 使得在 $G$ 中, 这些边 只与 $B$ 的顶点相关联. 因为 $\left\{x_{i-1}, y_{i-1}, z_{i-1}\right\} \cap B \neq \emptyset$, 所以可以假设 $x_{i-1} \in B$. 因为 $|B|=\frac{n-1}{2}$, 所以 $\left|F_{i-1} \cap E(G[A])\right| \geqslant 2$, 从而假设 $a_{1} a_{2}, a_{3} a_{4} \in F_{i-1} \cap E(G[A])$. 设 $G_{i-1}=$ $G-\left(F_{1} \cup \cdots \cup F_{i-2}\right), G_{i-1}^{\prime}=G_{i-1}-\left\{b_{1} b_{2}\right\}$, 和 $H_{i-1}^{\prime}=G_{i-1}^{\prime}-\left\{x_{i-1}, y_{i-1}\right\}$. 再设 $V_{4}=$ $\left\{x_{1}, y_{1}, z_{1}, \ldots, x_{i-2}, y_{i-2}, z_{i-2}\right\}$. 因为 $G$ 是正则图, 所以对于 $u \in V_{4}$, 有 $d_{G_{i-1}}(u)=\Delta\left(G_{i-1}\right)$, 对于 $u \in V\left(G_{i-1}\right) \backslash V_{4}$, 有 $d_{G_{i-1}}(u)=\Delta\left(G_{i-1}\right)-1=\delta\left(G_{i-1}\right)$, 因此 $\Delta\left(G_{i-1}\right)=\delta\left(G_{i-1}\right)+1$, 从而

$$
\Delta\left(G_{i-1}\right)-2 \leqslant \delta\left(G_{i-1}^{\prime}\right) \leqslant \Delta\left(G_{i-1}^{\prime}\right) \leqslant \Delta\left(G_{i-1}\right),
$$

且 $G_{i-1}^{\prime}$ 有至多两个度为 $\Delta\left(G_{i-1}\right)-2$ 的顶点. 因为 $B$ 是 $H_{i}$ 的一个独立集, 以及 $\left\{b_{1}, b_{2}, x_{i-1}\right\}$ $\subset B$, 所以, 在 $H_{i}$ 中, $x_{i-1}$ 与 $b_{1}$ 和 $b_{2}$ 都不相邻. 因为在 $G_{i-1}$ 中, $x_{i-1}$ 不与 $F_{i-1}$ 的边相关 联, 以及 $b_{1} b_{2} \in F_{i-1}$, 所以在 $G_{i-1}$ 中, $x_{i-1}$ 与 $b_{1}$ 和 $b_{2}$ 都不相邻. 因为 $G$ 是正则图, 所以

$$
\Delta\left(G_{i-1}\right)-3 \leqslant \delta\left(H_{i-1}^{\prime}\right) \leqslant \Delta\left(H_{i-1}^{\prime}\right) \leqslant \Delta\left(G_{i-1}\right) .
$$

设 $\Delta_{1}=\Delta\left(G_{i-1}\right)$, 则 $G_{i-1}^{\prime}$ 是 $\left(\Delta_{1}-2, \Delta_{1}\right)$-图, 且 $H_{i-1}^{\prime}$ 是 $\left(\Delta_{1}-3, \Delta_{1}\right)$-图. 如同引理 9 的情 形 1.3 的证明, $H_{i-1}^{\prime}$ 是 2-连通的. 若 $H_{i-1}^{\prime}$ 是非 Hamilton 图, 因为 $i-1 \geqslant\left\lfloor\frac{2 n}{9}\right\rfloor+1$, 所以

$$
\left|V_{4}\right|=3(i-2) \geqslant 3\left\lfloor\frac{2 n}{9}\right\rfloor>3\left(\frac{2 n}{9}-1\right)=\frac{2 n}{3}-3 .
$$

因此 $G_{i-1}$ 有至少 $\frac{2 n}{3}-3$ 个度为 $\Delta_{1}$ 的顶点, 那么 $G_{i-1}^{\prime}$ 满足引理 4 的假设 (b), 从而 $H_{i-1}^{\prime}$ 和 $G_{i-1}^{\prime}$ 满足引理 4 的假设, 于是存在 $V\left(H_{i-1}^{\prime}\right)$ 的一个子集 $A_{i-1}$ 使得 $\left|A_{i-1}\right|=m-1$ 并 且 $\omega\left(H_{i-1}^{\prime}-A_{i-1}\right)=m$. 设 $B_{i-1}=V\left(H_{i-1}^{\prime}-A_{i-1}\right)$, 则 $B_{i-1}$ 是 $H_{i-1}^{\prime}$ 的一个独立集并且 $\left|B_{i-1}\right|=m$.

若 $B \cap B_{i-1}=\emptyset$. 如同引理 9 的情形 1.3 , 我们可以得到矛盾, 因此 $B \cap B_{i-1} \neq \emptyset$. 如同 (12) 式, 我们有 (注意 $x_{i-1} \in B$ )

因为 $\left|A_{i-1}\right|=\frac{n-1}{2}-1$, 所以

$$
\left|A \cap A_{i-1}\right| \geqslant \delta\left(H_{i}\right)-1 \geqslant \frac{n}{3}+7
$$

$$
\left|B \cap A_{i-1}\right| \leqslant\left|A_{i-1}\right|-\left|A \cap A_{i-1}\right| \leqslant \frac{n-1}{2}-1-\left(\frac{n}{3}+7\right)=\frac{n}{6}-\frac{17}{2} .
$$

情形 1.3.1 若 $\left|A \backslash A_{i-1}\right| \leqslant 5$. 由引理 $6,\left|B \backslash A_{i-1}\right| \geqslant m-5$, 则

$$
\left|B \backslash\left(A_{i-1} \cup\left\{x_{i-1}, y_{i-1}\right\}\right)\right| \geqslant m-7 .
$$

因为 $B \backslash\left(A_{i-1} \cup\left\{x_{i-1}, y_{i-1}\right\}\right) \subset B_{i-1}$, 所以 $\left\{b_{3} b_{4}, \ldots, b_{27} b_{28}\right\}$ 包含至少 1 条边, 记为 $b_{3} b_{4}$, 使 得 $b_{3} b_{4} \in E\left(H_{i-1}^{\prime}-A_{i-1}\right)$, 这与 $B_{i-1}$ 是 $H_{i-1}^{\prime}$ 的独立集矛盾.

情形 1.3.2 若 $\left|A \backslash A_{i-1}\right| \geqslant 6$. 设 $p=\left|A \backslash A_{i-1}\right|$, 因为 $x_{i-1} \in B$, 所以 $x_{i-1} \notin A$, 因此, 由 (22) 式, 得

$$
e_{H_{i}}\left(A \backslash\left(A_{i-1} \cup\left\{x_{i-1}, y_{i-1}\right\}\right), B\right) \geqslant(p-1) \delta\left(H_{i}\right)-2\left(\delta\left(H_{i}\right)-3\right),
$$

从而至少存在一个顶点 $v \in A \backslash\left(A_{i-1} \cup\left\{x_{i-1}, y_{i-1}\right\}\right) \subset B_{i-1}$, 使得 (注意 $x_{i-1} \in B$ )

$$
e_{H_{i}}(\{v\}, B) \geqslant \frac{(p-1) \delta\left(H_{i}\right)-2\left(\delta\left(H_{i}\right)-3\right)}{p}
$$

1198 
由 (23) 式,

$$
\begin{aligned}
& =\frac{(p-3) \delta\left(H_{i}\right)}{p}+\frac{6}{p} \geqslant \frac{3}{6} \delta\left(H_{i}\right) \quad(\text { 因为 } p \geqslant 6, \text { 如同 }(10) \text { 式) } \\
& \geqslant \frac{n}{6}+4 .
\end{aligned}
$$

$$
\begin{aligned}
e_{H_{i}}\left(\{v\}, B \backslash A_{i-1}\right) & \geqslant e_{H_{i}}\left(\{v\}, B \backslash\left(B \cap A_{i-1}\right)\right) \\
& \geqslant\left(\frac{n}{6}+4\right)-\left(\frac{n}{6}-\frac{17}{2}\right)>12,
\end{aligned}
$$

则

$$
e_{H_{i}-\left\{x_{i-1}, y_{i-1}\right\}}\left(\{v\}, B \backslash\left(A_{i-1} \cup\left\{x_{i-1}, y_{i-1}\right\}\right)\right) \geqslant 10 .
$$

因为 $H_{i}-\left\{x_{i-1}, y_{i-1}\right\}$ 是 $H_{i-1}^{\prime}$ 的一个子图, 所以 $e_{H_{i-1}^{\prime}}\left(\{v\}, B \backslash\left(A_{i-1} \cup\left\{x_{i-1}, y_{i-1}\right\}\right)\right) \geqslant 10$. 又因为 $B \backslash\left(A_{i-1} \cup\left\{x_{i-1}, y_{i-1}\right\}\right) \subset B_{i-1}$ 以及 $v \in B_{i-1}$, 所以 $B_{i-1}$ 不是 $H_{i-1}^{\prime}$ 的独立集, 矛 盾.

因此, $H_{i-1}^{\prime}$ 是 Hamilton 图. 设 $C_{i-1}^{\prime}$ 是 $H_{i-1}^{\prime}$ 中的一个 Hamilton 圈, 且设 $F_{i-1}^{\prime}$ 是 $H_{i-1}^{\prime}$ 中的最大匹配, 使得 $F_{i-1}^{\prime} \subset E\left(C_{i-1}^{\prime}\right)$ 且 $z_{i-1}$ 不与 $F_{i-1}^{\prime}$ 中的边相关联.

设 $G_{i}^{\prime}=G-\left(F_{1} \cup \cdots \cup F_{i-2} \cup F_{i-1}^{\prime}\right)$ 和 $H_{i}^{\prime}=G_{i}^{\prime}-\left\{x_{i}, y_{i}\right\}$. 如同引理 9 的证明 (值得注 意的是, 在应用引理 4 时, 我们可以检验 $G_{i}^{\prime}$ 满足引理 4 的假设 (b)), 可得引理成立. 从而引 理被证明. 由引理 10 和 7 , 我们得到定理成立. 从而定理 1 得证.

定理 2 设 $G$ 是 $n$ 阶正则图且

则 $\chi_{T}(G) \leqslant d(G)+2$.

$$
d(G) \geqslant \frac{2 n}{3}+\frac{23}{6}
$$

证明 由定理 1 和文献 [14] 的结果, 定理 2 成立.

致谢对审稿专家提出的宝贵意见, 作者表示衷心的感谢!

\section{参考文献}

1 Behzad M. Graphs and their chromatic numbers. Doctoral thesis, Michigan State University, 1965

2 Vizing V G. Some unsolved problems in graph theory. Russ Math Surv, 23: 125-142 (1968)

3 Behzad M, Chartrand G, Cooper Jr J K. The colour numbers of complete graphs. J Lond Math Soc, 42: 225-228 (1967)

4 Chew K H, Yap H P. Total chromatic number and chromatic index of complete r-partite graphs. J Graph Theory, 16: 629-634 (1992)

5 Kostochka A V. The total coloring of a multigraph with maximal 4. Discret Math, 17: 161-163 (1977)

6 Kostochka A V. The total chromatic number of any multigraph with maximum degree five is at most seven. Discret Math, 162: 199-214 (1996)

7 Vijayaditya N. On total chromatic number of a graph, J Lond Math Soc, 3: 405-408 (1971)

8 Borodin O V. On the total coloring of planar graphs. J Reine Angew Math, 394: 180-185 (1989)

9 Yap H P. Total-colourings of graphs. Manuscript, 1989

10 Andersen L. Total colouring of simple graphs (in Danish). Master's thesis, University of Aalborg, 1993 
11 Sanders D P, Zhao Y. On total 9-coloring planar graphs of maximum degree seven. J Graph Theory, 31: 67-73 (1999)

12 Hilton A J W, Hind H R. The total chromatic number of graphs having large maximum degree. Discret Math, 117: 127-140 (1993)

13 Yap H P. Wang J F, Zhang Z. Total chromatic number of graphs of high degree II. J Aust Math Soc Ser A, 53: 219-228 (1992)

14 Xie D, He Z. The total chromatic number of regular graphs of even order and high degree. Discret Math, 300: 196-212 (2005)

15 Xie D, Yang W. The total chromatic number of graphs of even order and high degree. Discret Math, 271: 295-302 (2003)

16 Wang W. Total chromatic number of planar graphs with maximum degree ten. J Graph theory, 54: 91-102 (2006)

17 Wang Y, Shangguan M, Li Q. On total chromatic number of planar graphs without 4-cycles. Sci China Ser A-Math, 50: 81-88 (2007)

18 Erdös P, Pósa L. On the maximal number of disjoint circuits in a graph. Publ Math Debr, 9: 3-12 (1962)

19 Jackson B. Hamilton cycles in almost-regular 2-connected graphs. J Comb Theory Ser B, 57: $77-87$ (1993)

20 Dirac G A. Some theorems on abstract graphs. Proc London Math Soc, 2: 69-81 (1952) 